\title{
Automated morphological phenotyping using learned shape descriptors and functional maps: A novel approach to geometric morphometrics
}

Oshane O. Thomas ${ }^{1 *}$, Hongyu Shen ${ }^{2}$, Ryan L. Raaum ${ }^{3,4,5}$, William E.H.

Harcourt-Smith $^{3,4,5,6}$, John D. Polk ${ }^{1,7 \ddagger}$, Mark Hasegawa-Johnson ${ }^{2 \ddagger}$

1 Department of Anthropology, University of Illinois at Urbana-Champaign, Urbana, IL, USA

2 Department of Electrical and Compute Engineering, University of Illinois at Urbana-Champaign, Urbana, IL, USA

3 Department of Anthropology, Lehman College, Bronx, New York, USA

4 Department of Anthropology, CUNY Graduate Center, New York, New York, USA

5 New York Consortium in Evolutionary Primatology, New York, New York, USA

6 Department of Vertebrate Paleontology, American Museum of Natural History, New York, New York, USA

7 Department of Biomedical and Translational Sciences, University of Illinois at Urbana-Champaign, Urbana, IL, USA

$\ddagger$ These authors are joint senior authors on this work.

* othomas2@illinois.edu

\section{Abstract}

The methods of geometric morphometrics are commonly used to quantify morphology in a broad range of biological sciences. The application of these methods to large datasets is constrained by manual landmark placement limiting the number of landmarks and introducing observer bias. To move the field forward, we need to automate morphological phenotyping in ways that capture comprehensive representations of morphological variation with minimal observer bias. Here, we present Morphological Variation Quantifier (morphVQ), a shape analysis pipeline for quantifying, analyzing, and exploring shape variation in the functional domain. morphVQ uses descriptor learning to estimate the functional correspondence between whole triangular meshes in lieu of landmark configurations. With functional maps between pairs of specimens in a dataset we can analyze and explore shape variation. morphVQ uses Consistent ZoomOut refinement to improve these functional maps and produce a new representation of shape variation, area-based and conformal (angular) latent shape space differences (LSSDs). We compare this new representation of shape variation to shape variables obtained via manual digitization and auto3DGM, an existing approach to automated morphological phenotyping. We find that LSSDs compare favorably to modern 3DGM and auto3DGM while being more computationally efficient. By characterizing whole surfaces, our method incorporates more morphological detail in shape analysis. We can classify known biological groupings, such as Genus affiliation with comparable accuracy. The shape spaces produced by our method are similar to those produced by modern 3DGM and to auto3DGM, and distinctiveness functions derived from LSSDs show us how shape variation differs between groups. morphVQ can capture shape in an automated fashion while avoiding the limitations of manually digitized landmarks, and thus represents a novel and computationally efficient addition to the geometric morphometrics toolkit. 


\section{Author summary}

The quantification of biological shape variation has relied on expert placement of relatively small subsets of landmarks and their analysis using tools of geometric morphometrics (GM). This paper introduces morphVQ, a novel, automated, learning-based approach to shape analysis that approximates the non-rigid correspondence between surface models of bone. With accurate functional correspondence between bones, we can characterize the shape variation within a dataset. Our results demonstrate that morphVQ performs similarly to manual digitization and to an existing automated phenotyping approach, auto3DGM. morphVQ has the advantages of greater computational efficiency and while capturing shape variation directly from surface model representations of bone. We can classify biological shapes to the Genus level with comparable accuracy to previous approaches, and we can demonstrate which aspects of bone shape differ most between groups. The ability to provide comparable accuracy in a Genus level classification with features extracted from morphVQ further guarantees the validity of this approach.

\section{Introduction}

Biologists studying bone surface morphology often quantify shape using the landmarks and semilandmarks of Geometric Morphometrics (GM) [1-6]. Such quantification permits us to analyze the differences between bones with manually identified homologous or corresponding landmarks. With landmarks, we can study biological features' locations in geometric relation to each other, which provides opportunities to examine the patterning of complex morphological phenotypes $[4,7,8]$. Modern three-dimensional GM analysis pipelines begin with the manual collection of tens to hundreds of landmarks from digital representations of bone such as triangular meshes (polygon models). They end with a set of Procrustes-aligned coordinate shape variables that retain the geometric information contained within the data [5] (Fig 1). These shape variables, geometric measures of an object invariant to location, scale, and orientation, are the object of these analyses. They make it possible to ask research questions about the structuring of biological shape variation. Consequently, GM analyses of shape variation have increased in importance and remain indispensable in theoretical and mathematical biology [5, 8].

In implementing landmark-based GM, morphologists must make a host of decisions and compromises that limit the range of phenotypic variability one can capture [9]. Researchers must make informed sacrifices about which parts of morphology to study by choosing a fixed number of landmarks of various types to capture the geometric features we judge to be most important to our questions. Critically, we are required to decide what aspects of morphology must be measured and how to use landmarks to quantify that variation [9-11]. Landmarks reduce the inherent complexity of morphology down to a limited set of phenotypic structures. Hence, they cannot describe all aspects of a bone's shape. Although expertly chosen and theoretically grounded given the use-case, landmark configurations assume that the researcher has a priori knowledge of what morphological features are important, which is often not the case [9,12]. Gaining expert knowledge about phenotypic structures and then measuring them is often arduous, costly, and time-consuming. Notably, the landmarking process is prone to observer bias, and inter-and intra-observer error in identifying homologous landmark-based features can distort results $[8,9]$. As our questions begin to warrant (i) a comprehensive, unbiased measurement of morphology, (ii) quantification of more complex skeletal elements or structures without a priori assumptions about feature importance, and (iii) an ability to capture relevant shape variation for more extensive samples of complex 
Fig 1. Dataflow graphs of shape analysis pipelines used. Rounded rectangular objects represent data objects and arrows depict the procedures/algorithms operating on or producing them. All three analyses begin with triangular mesh models of bone. In (A), triangular mesh models are manually digitized. The configurations of corresponding/homologous points obtained are subjected to Generalized Procrustes analysis (GPA) to yield Procrustes pseudolandmark coordinate shape variables. (B) represents automated quantification using Auto3MGM. Farthest point sampling (FSP) is used to subsample triangular meshes. The Generalized Dataset Procrustes Framework (GDPF) of auto3DGM assigns correspondences and aligns subsampled shapes to a common coordinate system (rigid alignment). (C) outlines the proposed descriptor learning approach that builds on auto3DGM with learned non-rigid/deformable functional correspondences between aligned polygon models. These functional maps are used to estimate latent shape space differences that characterize morphological variation expressed as area-based and conformal operators. Note that the GDPF step here subsamples shape at a low resolution with only 128 and 256 pseudolandmarks for initial and final alignment steps, respectively. While not sufficient for capturing shape variation, low-resolution auto3DGM produces the rotation and translation information needed to rigidly align our polygon models.

biological shapes, researchers are increasingly looking to automated morphological phenotyping techniques as a solution $[8,13]$.

Despite the demand for automated morphological phenotyping methods, only a few algorithms exist. Thus far, the only approach to morphological phenotyping that does not require manual digitization and attempts to quantify morphology

comprehensively/exhaustively is auto3DGM [10]. This landmark-based algorithm represents each bone with a set of feature points uniformly and evenly sampled from each surface. It frames the task of aligning these 'pseudolandmarks' as an optimization problem. Automation hinges on the Iterative Closest Point-like alignment of configurations of pseudolandmarks that reasonably match corresponding points to each other [14]. auto3DGM substitutes modern 3DGM's sparse set of expert identified landmarks and semilandmarks for dense sets of algorithmically determined pseudolandmarks. These pseudolandmarks are aligned to yield Procrustes pseudolandmark coordinate shape variables much like those of modern 3DGM. While powerful, auto3DGM requires hundreds to thousands of pseudolandmark points to capture shape variation with adequate detail. This can be computationally costly and time consuming when sample sizes are large.

This study develops and validates a methodological first step towards automating morphological phenotyping and conducting morphometric analysis in the Functional Map (FM) Framework of Geometry Processing [15-17]. Ovsjanikov et al. 2012 present a procedure that relies upon functions defined on shapes, rather than points on them, to approximate their non-rigid or deformable correspondence. Correspondences between full triangular meshes or polygon models are expressed as linear operators between spaces of functions permitting the holistic study of differences in shape [16,17]. In modern 3DGM, an expert morphologist identifies a sparse set of corresponding or homologous points on each polygon mesh and registers them via Generalized Procrustes analysis (GPA). An automated FM-based approach estimates correspondences between whole geometric objects algorithmically. Given correspondences, we can characterize the shape variation and tackle a host of statistical shape analysis tasks. FM-based shape analyses are now being used to capture and analyze variation in synthetic and real shape collections [18-23]. This study extends these methods to the analysis of collections of biological shapes.

Our primary contribution is a novel learning-based approach to producing highly 
accurate FM correspondences between biological shapes directly from their polygon model geometry. We then use functional map network (FMN) analyses to characterize shape variability [16]. In addition to expressing correspondences between shapes, FMs can be manipulated to express shape differences [24]. Shape difference operators are descriptions of shape deformations encoded as changes in two inner products between functions. These linear operators capture the disparity between shapes and decompose it into area-based shape differences (local changes in the area of the object's surface) and conformal (angular) shape differences (local changes in the angles between vectors tangent to the surface) [17]. The vectors of measurements whose inner products are the area-based and conformal shape differences, respectively, can be viewed as shape variables, comparable to Procrustes aligned coordinates, since they capture useful geometry and are invariant to isometries [16]. For simplicity, we call this shape analysis pipeline Morphological Variation Quantifier (morphVQ).

We compare the conformal and area-based shape variables obtained from morphVQ to those obtained via manual digitization. We also compare them to shape variables produced by auto3DGM [10]. We assess the morphometric performance of our characterizations of shape by considering (i) how the shape spaces they form compare to those of modern 3DGM and auto3DGM, (ii) how accurately we can predict a priori biological groupings from them, and (iii) how between-group morphological differences obtained from them compare to those evidenced by modern 3DGM and auto3DGM. Given these performance criteria, we find that our characterization of shape variation is comparable in morphometric performance to representations obtained via manual digitization and auto3DGM.

\section{Results}

This study compares several morphometric analyses of the same 102 hominoid cuboids. The cuboid bone - often referred to as the hominoid foot's 'keystone' element - sits laterally in the primate midfoot. Biological anthropologists are interested in cuboid form because there is a robust evolutionary association between primate cuboid morphology, pedal joint function, and inferred locomotor behavior [25-34]. As such, researchers are interested in how cuboid shape and size covary with other morphological, functional, or behavioral phenotypes, as fossil cuboids can provide insights into the locomotor behaviors of extinct taxa. Automatically and exhaustively quantifying hominoid cuboid shape is a worthwhile real-world task for developing and evaluating our morphological phenotyping approach.

To establish a baseline for comparison, we capture two representations of hominoid cuboid shape using the expert identified landmarks and semilandmarks of modern 3DGM (see Methods and Materials for details) (Fig 1A). The first uses 21 type I-III landmarks placed on discrete features on each polygon mesh. The second captures proximal and distal cuboid facet shape using 130 sliding surface semilandmarks. These landmark configurations have been used in previous studies to quantify cuboid shape variation [35]. We consider them ground-truth representations for our series of comparisons because they are based on specific cuboid feature importance models.

\section{1 auto3DGM and rigid alignment}

auto3DGM plays two roles in this study. In its first role, auto3DGM generates Procrustes pseudolandmark coordinates based on dense subsamples of points from the surface of each shape in our collection (Fig 1B). These algorithmically obtained shape variables directly characterize shape variation, so they are retained for our comparisons. With auto3DGM, we find that a sufficiently adequate analysis of hominoid cuboid 
shape requires at least 512 pseudolandmarks to quantify surface shape well. With greater than 512 points and a moderately sized collection of shapes, this Iterative Closest Point-like procedure can become computationally prohibitive.

In its second role, auto3DGM is used to rigidly align our collection of polygon models to a global coordinate system. Our algorithm performs best when polygon models are approximately rigidly aligned as this avoids extrinsic symmetries that can't be disambiguated by our learning step [36]. auto3DGM's Generalized Dataset Procrustes Framework (GDPF) propagates rotation and translation information obtained during the alignment of pseudolandmarks to the polygon models themselves. We find that auto3DGM can produce useful rigid alignments between our hominoid polygon models in our collection with as few as 256 pseudolandmarks. While the Procrustes pseudolandmark shape variables from a low-resolution analysis don't characterize shape with enough surface detail, the aligned polygon models produced by the procedure are used as input to the descriptor learning step (Fig 1C).

\subsection{Descriptor learning}

Our descriptor learning model (Fig 2), is a variant of SURFMNet (see Methods and Materials for additional details). SURFMNet is a Siamese neural network that learns to improve upon precomputed spectral shape descriptors to produce more accurate functional correspondences between shapes [37]. Our model differs from SURFMNet in two ways: (i) it accepts geometric data derived from rigidly-aligned polygon models as input as opposed to precalculated spectral shape descriptors, and (ii) it uses a Harmonic Surface Network (HSN) as a feature extractor in place of a fully connected residual network. We choose HSN as our feature extractor due to its ability to produce rotation-invariant features from polygon model geometry, an essential property for our descriptors (see Methods and Materials for additional details) [38].

Fig 2. Deep Functional Maps network architecture demonstrating functional and soft P2P map estimation in both directions. We start with an initial pair of source and target shapes $S_{1}$ and $S_{2}$, respectively. $\Theta$ is a Siamese harmonic surface network, and $\Phi$ and $\Psi$ are the truncated Laplacian eigenbases for $S_{1}$ and $S_{2}$. Learned spatial descriptors are then projected to their corresponding bases to form $F$ and $G . C_{12}$ and $C_{21}$ are $70 \times 70$ functional maps (FMs) estimated in the forward and backward directions between source and target. On the far right are the recovered $\mathrm{P} 2 \mathrm{P}$ maps $T_{12}$ and $T_{21}$, respectively. In P2P maps, visual representation of correspondence is demonstrated between (homologous) features that have the same color.

Our network's FM layer estimates the forward and backward correspondences, $C_{12}$ and $C_{21}$, between a source shape $S_{1}$ and a target shape $S_{2}$. These functional maps are easily converted to dense P2P correspondences, $T_{12}$ and $T_{21}$. Our approach to descriptor learning is practical, with learned descriptors producing consistent functional maps for all pairs of hominoid cuboid shapes in our collection.

\subsection{Improving correspondences with Consistent ZoomOut}

The P2P maps $\left(T_{12}\right.$ and $\left.T_{21}\right)$ obtained with the learned descriptors contain artifacts. These artifacts are visible in the correspondences between pairs of shapes presented in Fig 3A.1 and 3A.2. Published studies using FM in computer graphics usually minimize such artifacts with some sort of post-processing refinement step $[20,39,40]$. We adopt the Consistent ZoomOut refinement technique, which iteratively and interchangeably optimizes the $\mathrm{P} 2 \mathrm{P}$ and functional maps at multiple scales given the initial functional maps $C_{12}$ and $C_{21}$. Compared to their counterparts in Fig 3A.2, the Consistent 
ZoomOut refined P2P maps in Fig 3B.2 achieve a smoother representation with fewer artifacts. Furthermore, we observe that these smoother representations are associated with increased bijective coverage as there is a $4 \%$ to $12 \%$ improvement when Consistent ZoomOut refinement is applied. Here bijective coverage refers to the ratio of the number of points in a source shape with a corresponding unique point on the target shape to the total number of points on that source shape. The Consistent ZoomOut refinement procedure hinges on a Limit Shape functional map network (FMN) analysis. Limit Shape characterizes shape variation to yield our area-based and conformal latent shape space differences (LSSDs) (see Methods and Materials section for more information) $[21,24,40]$.

Fig 3. Improving correspondence with Consistent ZoomOut. We compare maps generated by HSN ResUNet descriptor learning model to their Consistent ZoomOut-refined counterparts for four randomly chosen pairs of hominoid cuboids. Rows 3A.1 and 3A.2 show source and target shape pairs produced by our model, respectively. Rows 3B.1 and 3B.2 show the same source and target shape pairs after Consistent ZoomOut refinement. Between shape pairs, surface regions of the same color (green/purple/pink/yellow) are considered homologous. Black areas on source shapes indicate a lack of bijective coverage with its associated location on the corresponding target.

\subsection{Shape space comparisons}

In Fig 4, we visually compare shape spaces derived from three modes of hominoid cuboid shape quantification with individuals colored by genus. All six plots show distinctive groupings among hominoids. Hylobates and Homo separate well from all other groups in all shape spaces while Pan, Gorilla, and Pongo overlap to varying degrees. We use Pearson's $r$ to assess the correlation between the manually digitized 21-landmark representation (Fig 4A) - a proxy for a ground-truth measurement - and our other representations from sliding semilandmarks, auto3DGM, and LSSDs (Fig 4B-F). As expected, the highest correlation exists between the 21 manually-placed landmarks $4 \mathrm{~A}$ and the other manually digitized sliding semilandmarks $4 \mathrm{~B}$, $r(100)=0.639, p<0.001$. Of the representations derived from automated methods, the conformal LSSDs are most correlated with the 21-landmark representation, $r(100)=0.621, p<0.001$. The auto3DGM 512-pseudolandmark representation 4D and the area-based LSSDs $4 \mathrm{E}$ are only slightly less correlated at $r(100)=0.616$, and , $r(100)=0.618, p<0.001$, respectively. We also assessed the proportion of variance each hominoid group accounts for in each representation using the trace of each group's variance-covariance matrix as a measure of spread. We found no difference in the variance partitioning between representations, i.e., groups account for the same proportion of total variance in each set of shape variables.

Fig 4. Shape space differences between manually digitized and automated morphological quantification approaches. The principal component (PC) scores $\mathrm{PC} 1$ and $\mathrm{PC} 2$ plotted in $\mathbf{A}$ and $\mathbf{B}$ are from the Procrustes aligned coordinates of the manually digitized landmarks. $\mathbf{C}$ and $\mathbf{D}$ are based on Procrustes-aligned pseudolandmarks obtained from auto3DGM analyses with 256 and 512 points, respectively. $\mathbf{E}$ and $\mathbf{F}$ are PCs obtained from our LSSD characterization. $\mathbf{E}$ is a morphospace derived from area-based differences in hominoid cuboid shape, while $\mathbf{F}$ is based on conformal shape differences. 


\subsection{Classifying known biological groupings}

The six resulting PC projections are then classified by genus using four standard machine learning algorithms: K-means, Logistic Regression, Naive Bayes, and Linear Discriminant Analysis (LDA) (see Methods and Materials section for more information). The resulting accuracies (average and standard deviation across eleven folds (table 1). All representations perform well at genus classification. As expected, the first representation based on manually digitized landmarks performs best with each classification algorithm, LDA being the only exception. Our traditional 130-semilandmarks representation of cuboid shape yields the high K-means and Logistic regression accuracies; however, conformal LSSDs perform just as well across both classifiers. Overall, conformal LSSDs perform well across classifiers with accuracy greater than $90 \%$ in all experiments.

Table 1. Accuracies and standard deviations for hominoid group classification task

\begin{tabular}{ccccccccc}
\multirow{2}{*}{$\begin{array}{c}\text { Quantification } \\
\text { Mode }\end{array}$} & \multirow{2}{*}{ Representation } & \multicolumn{2}{c}{ K-Means Logistic Regression Naive Bayes } & \multicolumn{2}{c}{ LDA } \\
\cline { 3 - 8 } & & Accuracy & Accuracy & Std. & Accuracy & Std. & Accuracy & Std. \\
\hline Manual & Homologous landmarks (21 points) & 0.990 & 0.994 & 0.025 & 0.954 & 0.067 & 0.846 & 0.122 \\
digitization & Semilandmark patches (130 points) & 0.932 & 0.984 & 0.041 & 0.847 & 0.114 & 0.596 & 0.154 \\
\hline \multirow{2}{*}{ Automated } & auto3DGM pseudolandmarks (256 points) & 0.627 & 0.976 & 0.071 & 0.931 & 0.094 & 0.755 & 0.127 \\
measurement & auto3DGM pseudolandmarks (512 points) & 0.906 & 0.97 & 0.055 & 0.901 & 0.093 & 0.736 & 0.136 \\
& Area-based LSSDs (70x70 functional map) & 0.632 & 0.975 & 0.051 & 0.874 & 0.105 & 0.696 & 0.14 \\
& Conformal LSSDs (70x70 functional map) & $\mathbf{0 . 9 3 1}$ & $\mathbf{0 . 9 8 1}$ & $\mathbf{0 . 0 4 5}$ & $\mathbf{0 . 9 4 2}$ & $\mathbf{0 . 0 7 3}$ & $\mathbf{0 . 9 3 9}$ & $\mathbf{0 . 0 7 9}$
\end{tabular}

PCs corresponding to $95 \%$ of shape variation are used for each classification experiment (see Methods and Materials for details)

\subsection{Highlighting where variability happens}

We used the conformal and area-based LSSDs obtained to explore hominoid cuboid shape variability. Given our collection of shapes, the FMs obtained by our approach, an arbitrary FMN, and the LSSD shape variables, we can obtain shape variability across pairs of hominoid subgroups expressed as weighted distinctive functions projected directly on the bone surface (see Methods and Materials for details) [21]. These distinctive functions highlight the locations where intrinsic distortions deviate most from the average distortion between hominoid groups.

First, for visual reference, we generated polygon models of group-mean shape configurations for each GM-based approach (Fig 5). Using our approach, we can then show distinctive functions between groups highlighting where variability happens (Fig 6 and 7). We compared the relative locations of the most distinctive between-group differences detected from modern 3DGM and auto3DGM to those areas identified by our function-based analysis (summarized in table 2). Overall, we found that the surface regions highlighted by our FM-based shape analysis are the same cuboid surface regions that are most different between hominoid groups in modern 3DGM and auto3DGM analyses. For instance, the primary shape difference detected between Pan and Homo in both analyses occurs at the proximal facet's plantar-beak and along the distal portion of the medial aspect. 
Fig 5. Polygon models of hominoid cuboid group-mean shape

representations. A and $\mathbf{B}$ are polygon models based on landmark and semi-landmark patches where the vertices are manually digitized points. $\mathbf{C}$ shows pseudolandmark points (the vertices) with Delaunay triangulation. D features a randomly selected cuboid polygon model from each group for reference in the same orientation.

Fig 6. Weighted distinctive functions highlighting where regions are most variable in area-based distortion between hominoid groups. Dark red indicates the most variability, white the least. Rows: I Proximo-medial view; II Lateral; III Medio-distal; IV Dorso-distal.

Fig 7. Weighted distinctive functions highlighting where regions are most variable in conformal distortion on randomly selected pairs of hominoid cuboids. Dark red indicates the most variability in surface angulation, white the least. Rows: I Proximo-medial view; II Lateral; III Medio-distal; IV Dorso-distal.

\section{Discussion}

This work demonstrates that it is feasible to automate morphological quantification in the FM framework of geometry processing. We used our descriptor learning model to produce high-quality spectral shape descriptors and FM correspondences between our hominoid cuboid polygon models. With these correspondences, we characterized shape variation within the shape collection using Limit Shape-based statistical shape analysis [21]. We found that the conformal and area-based LSSD shape variables perform as well as, or better than those obtained from 3DGM and auto3DGM. Therefore, we demonstrate an efficient, automated solution to capturing shape variation. LSSD shape variables capture the common landscape populated by the collection of polygon models, and there is a well-defined notion of distance between polygon models in this shape space $[16,17]$.

An FM-based shape analysis pipeline allows us to automate and standardize morphological phenotyping without manually digitizing each bone. Expert observation, interpretation, and digitizing are no longer rate-limiting steps in morphometric analyses [10]. With functional correspondences estimated between whole triangular meshes, we can analyze shape variation comprehensively and exhaustively since we are not limited by the practical and representational limitations of a reduced set of digitized landmarks. We are now permitted to ask questions and test hypotheses about the structuring of morphological variation based on robust evidence with fewer assumptions about which shape features are essential to sample.

The similarities between our LSSD characterization and those representations based on manually-digitized landmarks and auto3DGM tell us that our method captures meaningful morphometric information. Based on visual clustering patterns, the shape spaces associated with our area-based and conformal LSSDs in Fig 4E and 4F, respectively, bear striking resemblances to the ones formed by manually digitized landmarks in Fig 4A and 4B. The between-specimen euclidean distances of our LSSD representation are highly correlated with landmark-based distances $(r=0.621)$, indicating that shape variation is structured similarly between these methods. The two shape representations can predict specimen genus affiliation with accuracies of comparable magnitude, telling us that our LSSDs encode between-group differences just as well as manually-digitized measures do. These similarities in variance structure and morphometric performance strongly suggest that our LSSD shape variables characterize the same biologically meaningful geometry captured by our specialized configuration of manually placed landmarks. 
bioRxiv preprint doi: https://doi.org/10.1101/2021.05.18.444628; this version posted May 18, 2021. The copyright holder for this preprint (which

was not certified by peer review) is the author/funder, who has granted bioRxiv a license to display the preprint in perpetuity. It is made available under aCC-BY 4.0 International license.

Table 2. Prominent between-group shape differences detected in representations from each analysis

\begin{tabular}{|c|c|c|c|c|c|}
\hline $\begin{array}{l}\text { Difference } \\
\text { Type }\end{array}$ & Representation & Pan-Gorilla & Pan-Hylobates & Pan-Homo & Pan-Pongo \\
\hline \multirow{3}{*}{$\begin{array}{l}\text { Group } \\
\text { Mean } \\
\text { Shape } \\
\text { Difference }\end{array}$} & $\begin{array}{l}\text { Type I-III } \\
\text { homologous } \\
\text { landmarks }\end{array}$ & $\begin{array}{l}\text { 1. Reduction in } \\
\text { plantar beak } \\
\text { size } \\
\text { 2. Broadening } \\
\text { of MT4 and } \\
\text { MT5 facets }\end{array}$ & $\begin{array}{l}\text { 1. Proximodistal } \\
\text { elongation of } \\
\text { the lateral side }\end{array}$ & $\begin{array}{l}\text { 1.Proximo-distal } \\
\text { elongation } \\
\text { 2. Size and } \\
\text { orientation of } \\
\text { facets } 3 \text {. } \\
\text { Plantar beak position }\end{array}$ & $\begin{array}{l}\text { 1. Negligible } \\
\text { differences detected. }\end{array}$ \\
\hline & $\begin{array}{l}\text { Proximal and } \\
\text { distal } \\
\text { semilandmark } \\
\text { patches }\end{array}$ & $\begin{array}{c}\text { 1. Negligible } \\
\text { differences detected. }\end{array}$ & $\begin{array}{l}\text { 1. Proximodistal } \\
\text { elongation } \\
\text { of the lateral side } \\
\text { 2. Size and } \\
\text { orientation } \\
\text { of facets }\end{array}$ & $\begin{array}{l}\text { 1.Proximo-distal } \\
\text { elongation } 2 \text {. Size } \\
\text { and orientation of } \\
\text { facets } 3 \text {, } \\
\text { Plantar } \\
\text { beak position }\end{array}$ & $\begin{array}{l}\text { 1.Plantar/lateral } \\
\text { section of proximal } \\
\text { facet } 2 . \text { Relative } \\
\text { rotation of proximal } \\
\text { and distal facets }\end{array}$ \\
\hline & $\begin{array}{c}\text { auto3DGM } \\
\text { pseudolandmarks }\end{array}$ & $\begin{array}{l}\text { 1. Deepening of } \\
\text { peroneus longus } \\
\text { groove on the } \\
\text { lateral side }\end{array}$ & $\begin{array}{l}\text { 1. Proximodistal } \\
\text { elongation of } \\
\text { the lateral side } \\
\text { 2. Mediolateral } \\
\text { narrowing } \\
\text { 3. Angular } \\
\text { changes between } \\
\text { proximal and } \\
\text { distal facets }\end{array}$ & $\begin{array}{l}\text { 1.Proximo-distal } \\
\text { elongation } \\
\text { 2. Medial edge } \\
\text { of 4th MT facet. } \\
\text { 3. Plantar beak of } \\
\text { proximal facet }\end{array}$ & $\begin{array}{c}\text { Negligible } \\
\text { differences detected. }\end{array}$ \\
\hline \multirow{2}{*}{$\begin{array}{c}\text { Variability } \\
\text { localization* }\end{array}$} & $\begin{array}{l}\text { Conformal } \\
\text { LSSDs }\end{array}$ & $\begin{array}{l}\text { 1. Lateral part } \\
\text { of the } \\
\text { proximal facet }\end{array}$ & $\begin{array}{l}\text { 1. Lateral aspect } \\
\text { of bone } \\
\text { (including peroneus } \\
\text { longus groove) }\end{array}$ & $\begin{array}{l}\text { 1. Medial and } \\
\text { dorsal edge } \\
\text { of } 4 \text { th MT facet } \\
\text { 2. The plantar beak } \\
\text { of the proximal } \\
\text { facet }\end{array}$ & $\begin{array}{l}\text { 1.Plantar/lateral } \\
\text { section of proximal } \\
\text { facet } 2 \text {. Lateral } \\
\text { aspect of bone } \\
\text { (including peroneus } \\
\text { longus groove) }\end{array}$ \\
\hline & $\begin{array}{l}\text { Area-based } \\
\text { LSSDs }\end{array}$ & $\begin{array}{l}\text { 1. Dorso-lateral } \\
\text { margins of } \\
\text { the proximal } \\
\text { facet }\end{array}$ & $\begin{array}{l}\text { 1. Lateral aspect } \\
\text { of bone } \\
\text { (including peroneus } \\
\text { longus groove) }\end{array}$ & $\begin{array}{l}\text { 1. Plantar beak } \\
\text { of proximal facet } \\
\text { 2. The medial } \\
\text { edge of the } \\
\text { 4th MT facet }\end{array}$ & $\begin{array}{l}\text { 1. Peroneus longus } \\
\text { groove }\end{array}$ \\
\hline
\end{tabular}

They are enumerated $(1,2$, etc.) in order of prominence. The first three shape rows describe landmark-based differences in group mean shape. The last two rows describe the locations of significant shape variability between groups as indicated by weighted highlighted functions (see Methods and Materials for additional details)

Morphologists find GM tools and methods appealing because they allow us to study shape differences and variability in ways that preserve geometry throughout an analysis $[4,5,8]$. With modern 3 DGM, we can visualize the morphological variation we capture as landmark displacements in a low-dimensional embedding of shape space. By preserving intrinsic geometry, our FM-based approach affords the same biological utility. Albeit differently, conformal and area-based LSSDs encode detailed information about the disparity or distortion between shapes under their estimated correspondence. With the distinctive functions derived from LSSDs, we can explore shape differences by visualizing where variability localizes between groups. Instead of deforming an anatomical model of bone or some mean-configuration of landmarks to illustrate shape 
differences in a morphospace, distinctive functions highlight the modes and regions of shape variation directly on polygon model surfaces. This approach differs from other shape analysis methods because it recognizes morphological regions by patterns of variability rather than mean difference. Thus, this approach has a more direct application to evolutionary studies that seek to quantify morphological variability to test evolutionary hypotheses.

In comparing different species or groups of specimens, it is common to investigate which aspects of shape allow discrimination. With distinctive functions, we can identify the surface regions where shape is most variable between groups. In particular, we can show the locations where area-based and conformal distortions deviate most from the average between-group distortion (Fig $4 \mathrm{E}$ and $4 \mathrm{~F}$ (i.e., 'where variability happens' on the surface [18]). We found that our distinctive functions highlight the same hominoid cuboid regions where our modern 3DGM and auto3DGM analyses detect significant between-group differences in shape, providing further evidence that the algorithm is detecting biologically meaningful shape information (table 2). With distinctive functions highlighting relevant variability, these methods permit automated detection of the regions or features that are morphologically distinctive for inter-group comparisons.

Here we find that learning spectral descriptor using an HSN feature extractor with auto3DGM rigid-aligned input polygon models leads to highly bijective FM correspondences with good coverage between all pairs of polygon models compared to either directly solving for correspondence using precomputed Wave Kernel Signature (WKS) descriptor or learning a better descriptor using FMNet or SURFMNet. This is because correspondence quality depends heavily on the geometric properties of the pointwise shape descriptor used to solve the problem. The WKS is a popular choice because it is multiscale, isometry invariant, easy to compute, and contains all intrinsic information at each point $[41,42]$. Despite its benefit, there are drawbacks to using WKS from real-world polygon model representations of bone (see S3 Fig). Though for different reasons, the well-documented sensitivity and specificity issues of WKS and HKS, respectively, can lead to poor correspondence quality, especially with a dataset of disparately obtained bone polygon models that differ drastically in shape and triangulation [43]. For instance, WKS descriptors yield high-quality bijective correspondences between similarly shaped Pan cuboids but produce poor correspondences between Pan and Hylobates cuboids as they are quite different in form. In contrast, our model encodes informative spectral descriptors that yield high-quality correspondences in both scenarios.

In summary, studies that characterize morphological phenotypes have relied on the analyses of manually digitized landmarks. Such analyses impose a priori constraints on which aspects of surface morphology can be captured, and an increasing body of evidence points to the fact that we need more than a few key traits to adequately characterize morphological variation $[10,12,44-46]$. We demonstrate that FM-based methods can automate comprehensive morphological quantification and provide a nuanced analysis of intrinsic shape variability. With efficient descriptor and correspondence learning, and FMN-based analysis tools like Limit Shape, we can make significant advances toward expanding the GM toolkit to include landmark-free analyses of biological shapes.

\section{Materials and methods}

\subsection{Data Acquisition and Preprocessing}

Our study sample consists of 102 triangular meshes obtained from laser surface scans of hominoid cuboid bones. These cuboids were from wild-collected individuals housed in 
the American Museum of Natural History, the National Museum of Natural History, the Harvard Museum of Comparative Biology, and the Field Museum. Hylobates, Pongo, Gorilla, Pan, and Homo are all well represented. Each triangular mesh is denoised, remeshed, and cleaned using the Geomagic Studio Wrap Software [47]. The resulting meshes vary in vertex-count/resolution from 2,000 - 390,000. Each mesh is then upsampled or decimated to an even 12,000 vertices using the recursive subdivisions process and quadric decimation algorithm implemented in VTK python, respectively [48-50].

\subsection{Expert measured and auto3DGM cuboid form quantification}

We used Stratovan Checkpoint Software [51] to quantify shape variation in two modes. The first configuration is of 21 well established type I-III landmarks placed on prominent points and facet margins, the second configuration consists of two semi-landmark patches placed on the proximal and distal articular facets only. The semilandmark patches in the second mode were anchored using 8 homologous landmarks for a total of 130 . These sets of landmark configurations were subjected to a generalized Procrustes analysis with semi-landmark sliding in the Geomorph $\mathrm{R}$ package $[3,6,52,53]$. The Procrustes-aligned coordinates from both were retained for further analysis.

For comparison, we used the auto3DGM R package to capture hominoid cuboid shape variation at two resolutions, with 256- and 512-pseudolandmarks,

respectively $[10,14]$. The lower resolution analysis was initialized with 150 subsampled points and the higher resolution was initialized with 256 points. The Procrustes-aligned pseudolandmarks obtained from these shape analyses are then subjected to a separate principal component analysis (PCA). The PC scores and eigenvalues from both are retained for further analysis. We also retain the aligned polygon models produced by the lower resolution analysis for our descriptor learning procedure.

\subsection{Functional maps and descriptor learning}

Using MATLAB, we rescaled each auto3DGM aligned polygon model to unit area. We discretized each model using the cotangent weighting scheme to yield stiffness and mass matrices that are then used to compute the Laplace-Beltrami Operator (LBO) via eigendecomposition [54]. We retained the LBO and the raw polygon model geometry (vertices and triangles) for descriptor learning in the FM-based framework in our proposed model.

We use the FM framework because there are several advantages to solving the correspondence problem in the functional domain, especially with the LBO as a basis. The LBO, a generalization of the Fourier analysis on Riemannian manifolds, is the eigenbasis of choice as it is invariant to isometric transformations, and it is well suited for continuous maps between geometric objects $[15,18]$. With the LBO as a basis we can use FM-based tools to efficiently transfer functions from a source to a target shape to avoid manipulating pointwise correspondences. The FM-based correspondence problem is linear and easy to optimize compared to the non-convex correspondence problem faced when points are considered. Using a truncated LBO with as few as 70 eigenfunctions reduces the dimensionality of the problem significantly without much loss to correspondence quality [17].

Given bones as source and target shapes, denoted by $S_{1}$ and $S_{2}$, the framework proposes the following general steps to calculate the functional maps:

1. First, we obtain $k_{1}$ and $k_{2}$ number of basis functions (LBO eigenfunctions) on the source and target shapes, respectively. We then project sets of precomputed shape descriptors (e.g., pointwise features that encode the local and global geometric 
properties of each surface) onto their respective bases to yield coefficients denoted $A$ and $B$.

2. The functional map framework focuses on the following equation to solve for $C$, the map that preserves the point-to-point map correspondence between the shapes:

$$
\mathrm{C}_{\mathrm{opt}}=\arg \min _{\mathrm{C}} E_{\mathrm{desc}}(\mathrm{C})+\alpha E_{\mathrm{reg}}(\mathrm{C})
$$

Here are the eigenvalue matrices of the two sets of basis functions:

$$
\begin{gathered}
E_{\text {desc }}(\mathrm{C})=\|\mathrm{CA}-\mathrm{B}\|^{2} \\
E_{\mathrm{reg}}(\mathrm{C})=\left\|\mathrm{C} \Lambda_{1}-\Lambda_{2} \mathrm{C}\right\|^{2}
\end{gathered}
$$

3. Once the functional map is obtained, standard implementations then recover the point-to-point map by nearest neighbor search. Standard implementations improve the quality of the point-to-point map using post-processing refinement tools such as Bijective Continuous Iterative Closest Point (BCICP) or Kernel Matching [20,39]. These post-processing steps are bypassed in our implementation, in favor of refinement using the Limit-Shape based Consistent Zoomout method (see Implementation for details)

While estimating FMs in this way is efficient and practical for whole polygon models, it can be quite sensitive to the type of shape descriptor used. There are multiple types of point-based descriptors of shape that provide initial points for mapping shapes to each other in the FM framework. These task-specific descriptors are designed to have specific geometric properties depending on their use case [43]. For example, spectral shape descriptors from solutions to the heat and wave equations are invariant to isometric transformations and are potent descriptors of intrinsic geometry. Such spectral descriptors are the current state-of-the-art $[41,55,56]$. Several studies show that it is possible to learn spectral descriptors directly and that learned spectral descriptors perform better in practice $[37,43,57,58]$.

The best performing descriptor learning models, such as FMNet and SURFMNet, are based on Siamese neural networks that transform pairs of shape descriptors into new ones to improve functional correspondence [37,59]. Despite being learning-based, they still adhere to the three step FM-pipeline previously described. With precomputed SHOT or WKS descriptors as input, these models use a deep residual neural network (ResNet) with shared weights to produce new features that are then projected into their respective eigenbases to create descriptors. FMNet and SURFMNet then use these new descriptors to estimate improved functional correspondences between shapes.

\subsection{Learning intrinsic features from surfaces}

Instead of using precomputed SHOT or WKS descriptors as the functions in a functional maps framework, several recent methods focus on learning spectral descriptors via the functional characterization of the vertices/point clouds of polygon models $[36,60]$. These approaches use specialized neural network architectures (e.g., PointNet++) or novel convolutional kernels (GCCN, MDGCN. KP-Conv, Metric-based 
Spherical Convolutions, etc. [61-64]) capable of exploiting the geometric features of point-clouds or triangular meshes. These descriptor learning models replace the Siamese ResNet architecture of FMNet with spatial feature extractors that have been implemented in a Siamese way [60]. Given the unstructured point clouds, they create new features that are invariant to translation, rotation, and point order. Just as in FMNet, these features are then projected into the eigenbasis to form the spectral descriptors needed to estimate functional correspondence. Point-cloud-based feature extraction approaches such as these yield higher quality correspondences compared to their precomputed descriptor-based counterparts.

Despite the advantages, extracting features of sufficient quality for functional characterization is difficult. Existing methods either (i) suffer from poor expressivity or (ii) are too sensitive to differences in polygon model connectivity, or (iii) they don't produce rotation-invariant features in a manner that is conducive to learning spectral descriptors. In this study, we craft spectral descriptors in a similar point-cloud-based fashion, but we use a Harmonic Surface Network (HSN) as a feature extractor [38]. HSN-based feature extractors produce highly expressive intrinsic features that are strongly locally-aligned. Compared to PointNet++, an HSN feature extractor encodes rotation-invariant features in a way that is more forgiving of arbitrary differences in pose ( $\mathrm{SO} 3$ rotations) between a source and a target polygon model. This is usually the case for real-world pairs of bone meshes which may be poorly aligned, if at all. In practice, we find that descriptors from HSN-based features consistently yield highly bijective FMs with detailed and accurate pointwise correspondences.

\subsection{Implementation}

\subsubsection{Deep HSN UResNet feature extractor}

We adopt the deep U-ResNet HSN architecture used for shape classification and segmentation introduced in [38]. Our U-ResNet architecture is deeper with eight stacks of ResNet blocks and three levels of pooling and unpooling layers (see S1 Fig) ResNet blocks are unchanged, each containing two HSN convolutional layers and a residual connection. Like Wiersma et al., we configure the network with rotation order streams: $\mathrm{M}=0$ and $\mathrm{M}=1$, where the former enforces rotation invariance and the latter, equivariance. For our model configuration, we use 16-, 24-, 32-, and 48- units at each scale of the deep U-ResNet. Pooling and unpooling are done in the same way via parallel transport, but with pooling ratios $1,0.5,0.25$, and 0.1 starting with a radius of 0.1 that grows by a factor of 2 at each scale. The 48-unit output produced by our last ResNet block then passes through the unpooling and reverse convolution layers of the U-net. This results in a 16-dimensional vector at each node of the original triangular mesh, which is then transformed to a 300-dimensional vector by a densely connected layer with ReLu activation. This HSN feature extractor was implemented using code from Wiersma et al., 2020 which was retrieved from https://github.com/rubenwiersma/hsn.

\subsubsection{Functional map layer and unsupervised loss}

The Siamese HSN feature extractor produces 300-dimensional spatial embeddings at each vertex in our source and target shapes. We projected these embedding into their respective LBO eigenbases to form spectral descriptors, which are then passed to a fully differentiable functional map layer that computes the functional maps $\mathrm{C}_{12}$ and $\mathrm{C}_{21}$ (in both directions) between the source and target shapes. After obtaining $\mathrm{C}_{12}$ and $\mathrm{C}_{21}$, we use the axiomatic SURFMNet loss defined in Roufosse et al. 2019 w.r.t. the model weights to optimize for the best $\mathrm{C}_{12}$ and $\mathrm{C}_{21}$. This is done directly in the functional 
domain.

This unsupervised loss consists of 4 regularization terms (or penalties) that assess the structural properties of the functional maps obtained from the FM-layer [ [37]. The first penalty, E1, enforces bijectivity by requiring that $\mathrm{C}_{12}$ and $\mathrm{C}_{21}$ be inverses of each other, which ensures that the composition of the two maps is an identity map. The second penalty, E2, constrains orthogonality. This condition preserves the local area of the two input shapes in the P2P map when converting back from the two functional maps. The third, E3, is a well-known regularizer in the functional map pipeline because it enforces commutativity with the Laplacian $[15,65]$. The fourth, E4, guarantees that the learned correspondences in the form of functional maps directly arise from the P2P map. Specifically, it means both functional maps are commutable w.r.t. the reduced bases of the descriptors of the corresponding source and target shapes. The FM layer and the unsupervised losses were implemented using a PyTorch Geometric [66] library retrieved from https://github.com/pvnieo/SURFMNet-pytorch.

\subsubsection{Training Scheme}

Following Wiersma et al., 2020, we precomputed the logarithmic maps, weights, and multi-scale radius graphs needed for HSN feature extraction from each auto3DGM aligned polygon model. We also retain the point-cloud/vertices of each aligned model as input to our network. Each model was trained using the ADAM optimizer with a learning rate of 1x10-3 in a data-parallelized manner [67]. Models are trained on random pairs of shapes drawn from the set of all pairwise comparisons without replacement. An epoch is complete when each pair of shapes in the collection has been seen once. This is repeated until convergence in a self-supervised manner with no validation set. At convergence, each pair of shapes is processed to produce a full set of shape descriptors, and a pair of functional maps from source to target shape and the reverse. These are all retained for further analysis. These models were trained with a batch size of one on a NVIDIA Tesla K80 GPU core.

\subsection{Limit shape and Consistent ZoomOut}

The Limit Shape is comparable or analogous to the Procrustes mean shape used in landmark-based GM analyses. It is computed with respect to a generalized eigendecomposition of the functional maps that capture the differences among shapes in a collection [21]. Specifically, if the LBO of each shape is discretized as its inverse mass matrix times its stiffness matrix, then the limit shape is defined to be the shape whose LBO is given by the arithmetic averages of the mass and stiffness matrices. Limit Shape analysis finds a set of canonical consistent latent bases (CCLBs) for each shape such that the functional map between any pair of shapes is represented by the projection of one shape onto its CCLB, followed by expansion onto the CCLB of the other. Using the CCLBs, it is possible to define the area-based and conformal shape variables, of any particular shape in the collection, respectively, to be the area-based and conformal shape differences between the particular shape and the limit shape. By having the shape differences between limit shape and every shape in the dataset, a joint mapping between shape $S_{i}$ and shape $S_{j}$ can be formulated as two consecutive mappings measuring the differences between shape $S_{i}$ and the limit shape and between shape $S_{j}$ and the limit shape.

Consistent ZoomOut is a method for obtaining improved point-to-point mappings between the shapes in a collection. It considers as input a set of shapes and their potentially low-quality pairwise point-to-point maps in a connected functional map network (FMN) and outputs point-to-point maps that have been improved. Specifically, the method proposes an iterative update of the functional maps $\left(\mathrm{C}_{i j}\right)$ matrices at 
different scales for a more accurate point-to-point mapping, especially when the input shapes are of different scales. The approach also considers the previous Limit Shape method as an intermediate step to produce consistent point-to-point mappings in each of the iterations. The Limit Shape method's CCLB matrices [40] provide canonically consistent bases across different shape inputs, normalizing for shape-dependent inconsistencies in the LBO. Taking advantage of these techniques, we input the learned functional maps $\mathrm{C}_{i j}$ from the HSN-based descriptor learning model to the Consistent ZoomOut algorithm and obtain the point-to-point mappings and the shape differences, two major components for our study. Limit Shape was implemented using MATLAB code retrieved from https://github.com/ruqihuang/SGP2020_ConsistentZoomOut.

\subsection{A priori biological group classification tasks}

After obtaining the shape differences through Consistent ZoomOut and Limit Shape, we perform the following classification/clustering tasks to evaluate whether our proposed method is able to extract morphological features that capture and characterize the shape differences present in a collection of hominoid cuboids. In particular, we fit models with six different types of input data. The first two representations of hominoid cuboid shape are based on procrustes aligned landmark configurations of 21 type I-III and 130 sliding semilandmarks, respectively. The third and fourth representations are auto3DGM procrustes pseudolandmarks quantified with -256 , and -512 points respectively. The fifth and sixth representations are conformal and area-based LSSDs from our morphVQ analysis. We then calculate the principal components given the six different inputs and choose the number of PCs to cover $95 \%$ of the total variance. The PCs are the final inputs for the Genus classification tasks. Specifically, we consider the following four tasks, three of which are supervised classifications and one is based on unsupervised clustering. Namely, we choose Logistic regression, Linear Discriminant Analysis, Naive Bayes as our supervised classifiers. We use K-means to be our unsupervised clustering algorithm. Comparing the performance of PCs with six different inputs on these four algorithms provides insight into which types of the extracted feature are better in identifying morphological differences. Here we provide a brief summary of the four classification (supervised/unsupervised) algorithms:

\subsubsection{Logistic regression}

Logistic regression is a generalized form of linear regression, which regresses the log odds of the probability of choosing one class over the other class. To characterize such correlations, a linear regression model is simply transformed by a sigmoid function. The transformed function models $p(y \mid x)$, where $x$ refers to the input features (in our case $\mathrm{PCs})$ and $y$ is the class label. Refer to equation for details. Where $a^{T}$ and $b$ refer to the parameters ("slope" and bias) in the logistic regression.

$$
\log \frac{p(y=1 \mid x)}{p(y=0 \mid x)}=a^{T} x+b
$$

\subsubsection{Linear Discriminant Analysis (LDA)}

LDA is a linear classifier aimed to find a projection direction such that the input features, once projected with respect to the given direction, have the maximum variance between classes and minimum variance within each of the classes. 


\subsubsection{Naive Bayes}

This is one of the simplest classifiers under the assumption that each of the feature dimensions is independent. Taking advantage of Bayes' Rule: $p(y \mid x) \sim p(x \mid y) p(y)$, we further decompose this equation into the multiplication of the independent likelihoods with the probability for the class $y: p(x \mid y)=\prod_{i=1}^{n}\left(x_{i} \mid y\right)$, where $\mathrm{n}$ denotes the dimension of the inputs. By applying the "maximum a posteriori (MAP)" classification rule, we are able to find the classification given the input features by: $y=\arg \max _{k} p(y=k) \prod_{i=1}^{n}\left(x_{1} \mid y=k\right)$, where $\mathrm{k}$ denotes the class index.

\subsubsection{K-means}

Different from previous approaches, K-means is an unsupervised approach that separates input data into different clusters based on the distances between each point in the input data and the means of each of the clusters. Here $K$ is a pre-specified hyperparameter that controls the number of clusters in the algorithm. Once it is confirmed (in our case it is the number of species), the algorithm will iteratively update the classifications of points in the input data and the cluster means to minimize the following objective: $\arg \min _{S} \sum_{i=1}^{k} \sum_{x \in S_{i}}\left\|x-\mu_{i}\right\|^{2}$, where $S=S_{1}, S_{2}, \ldots, S_{k}$ and each $S_{i}(i=1,2, \ldots, k)$ denotes the set of inputs to class $i . i$ refers to the mean of the inputs in $S_{i}$. And $k$ is a predefined hyperparameter for the number of classes.

To properly evaluate the performance of the above tasks, we apply 11-fold cross validation on the input shape differences. All results are summarized in Fig 1 in S2 Fig with standard deviations indicated according to the cross validation results.

\section{Supporting information}

S1 Fig. Harmonic surface network feature extractor used for descriptor learning. Adapted from Wiersma et. al., 2020. On the left is the U-ResNet structure with three pooling levels, on the right, is a detailed description of the ResNet block.

S2 Fig. Spectral descriptors learned by our model lead to accurate functional maps. The first five dimensions of the spectral descriptor learned with our HSN feature extractor. Corresponding/homologous regions on Hylobates source (above) and Pan target (below) shapes are similar in color. These learned descriptors yield high-quality FM correspondences.

S3 Fig. Correspondence quality is improved by learning descriptors compared to direct optimization with orientation operator. Point-to-point maps between source Pan shape and Hylobates target shape (left to right) obtained via direct optimization using WKS (above) and our learned spectral descriptor (below) [20]. Source and target shapes are remeshed to 12,000 vertices in both experiments. WKS were computed using 200 Laplace-Beltrami eigenfunctions.

S4 Fig. LSSDs lead to similar genus classification accuracies with logistic regression. Confusion matrices of multinomial logistic regression prediction for each representation of hominoid cuboid shape. Fig (A) and (B) use principal components of Procrustes-aligned semilandmark patches and pseudolandmarks generated by auto3DGM as independent variables, respectively. Independent variables in (C) and (D) are principal components of conformal and area-based LSSDs, respectively. PCs account for $95 \%$ shape variance for each representation, and stratified K-Fold cross-validation provides accuracies with standard deviations in parentheses. 


\section{Acknowledgments}

We would like to thank the collections managers at the American Museum of Natural History, National Museum of Natural History, Field Museum, and Harvard Museum of Comparative Zoology for access to mammalian osteological collections. We thank Cris Hughes and Lyle Konigsberg for academic guidance, and the Department of Anthropology for financial support. We would also like to thank Camille Goudeseune and Travis Ross of the Beckman Institute for their advice and guidance.

\section{References}

1. Gower JC. Generalized procrustes analysis. Psychometrika. 1975;40(1):33 51. doi:10.1007/bf02291478.

2. Dryden IL, Mardia KV. Statistical shape analysis: with applications in R. vol. 995. John Wiley \\& Sons; 1991.

3. Mitteroecker P, Gunz P. Advances in Geometric Morphometrics. Evolutionary Biology. 2009;36(2):235 247. doi:10.1007/s11692-009-9055-x.

4. Adams DC, Rohlf FJ, Slice DE. A field comes of age: geometric morphometrics in the 21 century Introduction. Italian Journal of Mammalogy. 2012;1(24):7. doi:10.4404/hystrix-24.1-6283.

5. Slice DE. Modern Morphometrics in Physical Anthropology. Springer Science \& Business Media. Springer Science \& Business Media; 2006.

6. Rohlf FJ, Slice D. Extensions of the Procrustes Method for the Optimal Superimposition of Landmarks. Systematic Biology. 1990;39(1):40 59. doi:10.2307/2992207.

7. Cardini A, Joy A. On growth and form in the "computer era": from geometric to biological morphometrics. Italian Journal of Mammalogy. 2013;1(24):5. doi:https://doi.org/10.4404/hystrix-24.1-8749.

8. Hallgrimsson B, Percival CJ, Green R, Young NM, Mio W, Marcucio R. Morphometrics, 3D Imaging, and Craniofacial Development. Current Topics in Developmental Biology. 2015;115:561-597. doi:10.1016/bs.ctdb.2015.09.003.

9. Macleod N. On the Use of Machine Learning in Morphometric Analysis. 4th International Symposium on Biological Shape Analysis (ISBSA). 2017; p. 134-171. doi:10.1142/9789813225701_0008.

10. Boyer DM, Puente J, Gladman JT, Glynn C, Mukherjee S, Yapuncich GS, et al. A New Fully Automated Approach for Aligning and Comparing Shapes. The Anatomical Record: Advances in Integrative Anatomy and Evolutionary Biology. 2014;298(1):249 276. doi:10.1002/ar.23084.

11. MacLeod N, Benfield M, Culverhouse P. Time to automate identification. Nature. 2010;467(7312):154 155. doi:10.1038/467154a.

12. Houle D, Govindaraju DR, Omholt S. Phenomics: the next challenge. Nature reviews Genetics. 2010;11(12):855 866. doi:10.1038/nrg2897.

13. Li M, Cole JB, Manyama M, Larson JR, Liberton DK, Riccardi SL, et al. Rapid automated landmarking for morphometric analysis of three-dimensional facial scans. Journal of Anatomy. 2017;230(4):607 618. doi:10.1111/joa.12576. 
14. Puente J. Distances and algorithms to compare sets of shapes for automated biological morphometrics; 2013.

15. Ovsjanikov M, Ben-Chen M, Solomon J, Butscher A, Guibas L. Functional maps: a flexible representation of maps between shapes. ACM Transactions on Graphics (TOG). 2012;31(4):30. doi:10.1145/2185520.2185526.

16. Ovsjanikov M, Corman E, Bronstein M, Rodolà E, Ben-Chen M, Guibas L, et al. Computing and processing correspondences with functional maps. 2016; p. 9. doi:10.1145/2988458.2988494.

17. Ovsjanikov M, Corman E, Bronstein M, Rodolà E, Ben-Chen M, Guibas L, et al. Computing and processing correspondences with functional maps. 2017; p. 5. doi:10.1145/3084873.3084877.

18. Ovsjanikov M. A Functional View of Geometry Processing; 2016.

19. Ren J, Schneider J, Ovsjanikov M, Wonka P. Joint Graph Layouts for Visualizing Collections of Segmented Meshes. IEEE Transactions on Visualization and Computer Graphics. 2017;24(9):2546-2558. doi:10.1109/tvcg.2017.2751473.

20. Ren J, Poulenard A, Wonka P, Ovsjanikov M. Continuous and orientation-preserving correspondences via functional maps. ACM Transactions on Graphics (TOG). 2019;37(6):248. doi:10.1145/3272127.3275040.

21. Huang R, Achlioptas P, Guibas L, Ovsjanikov M. Latent Space Representation for Shape Analysis and Learning. 2018;

22. Huang R, Rakotosaona MJ, Achlioptas P, Guibas L, Ovsjanikov M. OperatorNet: Recovering 3D Shapes From Difference Operators. 2019;.

23. Melzi S, Ren J, Rodolà E, Wonka P, Ovsjanikov M. ZoomOut: Spectral Upsampling for Efficient Shape Correspondence. 2019;.

24. Rustamov RM, Ovsjanikov M, Azencot O, Ben-Chen M, Chazal F, Guibas L. Map-based exploration of intrinsic shape differences and variability. ACM Transactions on Graphics (TOG). 2013;32(4):72. doi:10.1145/2461912.2461959.

25. Aiello L, Dean C. An Introduction to Human Evolutionary Anatomy. Academic Press. Academic Press; 1990.

26. Lewis OJ. The joints of the evolving foot. Part I. The ankle joint. Journal of Anatomy. 1980;130(Pt 3):527 543.

27. Lewis OJ. The joints of the evolving foot. Part III. The fossil evidence. Journal of Anatomy. 1980;131(Pt 2):275 298.

28. Lewis OJ. The joints of the evolving foot. Part II. The intrinsic joints. Journal of Anatomy. 1980;130(Pt 4):833 857.

29. Morton DJ. Evolution of the human foot I. American Journal of Physical Anthropology. 1922;V(4):305 336. doi:10.1002/ajpa.1330050409.

30. Morton DJ. Evolution of the human foot II. American Journal of Physical Anthropology. 1924;7(1):1 52. doi:10.1002/ajpa.1330070114.

31. Harcourt-Smith WEH, Aiello LC. Fossils, feet and the evolution of human bipedal locomotion. Journal of Anatomy. 2004;204(5):403 416.

doi:10.1111/j.0021-8782.2004.00296.x. 
32. Bojsen-Møller F. Calcaneocuboid joint and stability of the longitudinal arch of the foot at high and low gear push off. Journal of Anatomy. 1979;129(Pt 1):165 176.

33. DeSilva JM. Revisiting the "midtarsal break". American Journal of Physical Anthropology. 2010;141(2):245 258. doi:10.1002/ajpa.21140.

34. DeSilva JM, Gill SV. Brief communication: A midtarsal (midfoot) break in the human foot. American Journal of Physical Anthropology. 2013;151(3):495 499. doi:10.1002/ajpa.22287.

35. Harcourt-Smith W. Form and function in the hominoid tarsal skeleton. PhD Dissertation. 2002; University College London.

36. Sharma A, Ovsjanikov M. Weakly Supervised Deep Functional Map for Shape Matching. arXiv. 2020;.

37. Roufosse JM, Sharma A, Ovsjanikov M. Unsupervised Deep Learning for Structured Shape Matching. 2018;

38. Wiersma R, Eisemann E, Hildebrandt K. CNNs on surfaces using rotation-equivariant features. ACM Transactions on Graphics. 2020;39(4). doi:10.1145/3386569.3392437.

39. Lähner Z, Vestner M, Boyarski A, Litany O, Slossberg R, Remez T, et al. Efficient Deformable Shape Correspondence via Kernel Matching. 2017;.

40. Huang R, Ren J, Wonka P, Ovsjanikov M. Consistent ZoomOut: Efficient Spectral Map Synchronization. Computer Graphics Forum. 2020;39(5):265-278. doi:10.1111/cgf.14084.

41. Aubry M, Schlickewei U, Cremers D. The Wave Kernel Signature: A Quantum Mechanical Approach to Shape Analysis. 2011 IEEE International Conference on Computer Vision Workshops (ICCV Workshops). 2011;1:1626-1633. doi:10.1109/iccvw.2011.6130444.

42. Sun J, Ovsjanikov M, Guibas L. A Concise and Provably Informative Multi-Scale Signature Based on Heat Diffusion. Computer Graphics Forum. 2009;28(5):1383-1392. doi:10.1111/j.1467-8659.2009.01515.x.

43. Litman R, Bronstein AM. Learning Spectral Descriptors for Deformable Shape Correspondence. IEEE Transactions on Pattern Analysis and Machine Intelligence. 2014;36(1):171-180. doi:10.1109/tpami.2013.148.

44. Houle D. Colloquium papers: Numbering the hairs on our heads: the shared challenge and promise of phenomics. Proceedings of the National Academy of Sciences of the United States of America. 2010;107 Suppl 1(suppl_1):1793 1799. doi:10.1073/pnas.0906195106.

45. Pomidor BJ, Makedonska J, Slice DE. A Landmark-Free Method for Three-Dimensional Shape Analysis. PLOS ONE. 2016;11(3):e0150368. doi:10.1371/journal.pone.0150368.

46. Boyer DM, Lipman Y, Clair ES. Algorithms to automatically quantify the geometric similarity of anatomical surfaces. Proceedings of the National Academy of Science; 2011. 
47. Cheng Sy, Wu Wt, Yang Xr, Zhang H, Zhang Xw. Rapid surfacing reconstruction based on Geomagic Studio software [J]. Modern Manufacturing Engineering. $2011 ; 1$.

48. Loop CT. Smooth Subdivision surfaces based on triangles; 1987.

49. Garland M, Heckbert PS. Surface simplification using quadric error metrics. Proceedings of the 24th annual conference on Computer graphics and interactive techniques. 1997; p. 209-216. doi:10.1145/258734.258849.

50. Wills G. Visualization toolkit software: Visualization toolkit software. Wiley Interdisciplinary Reviews: Computational Statistics. 2012;4(5):474-481. doi:10.1002/wics.1224.

51. Stratovan. Stratovan Checkpoint; 2018. Available from: https://www.stratovan.com/products/checkpoint.

52. Collyer ML, Adams DC. RRPP: Linear Model Evaluation with Randomized Residuals in a Permutation Procedure, R package version 0.5.2. 2020;.

53. Zelditch ML, Swiderski DL, Sheets HD. Geometric morphometrics for biologists: a primer; 2012.

54. Meyer M, Desbrun M, Schröder P, Barr AH. Discrete Differential-Geometry Operators for Triangulated 2-Manifolds. In: Visualization and Mathematics III. Berlin, Heidelberg: Springer Berlin Heidelberg; 2003. p. 35-57.

55. Melzi S. Local Geometry Processing for Deformations of Non-Rigid 3D Shapes; 2018.

56. Bronstein MM, Bruna J, LeCun Y, Szlam A, Vandergheynst P. Geometric deep learning: going beyond Euclidean data. 2016;doi:10.1109/msp.2017.2693418.

57. Halimi O, Litany O, Rodolà E, Bronstein A, Kimmel R. Self-supervised Learning of Dense Shape Correspondence. 2018;

58. Bronstein AM. Spectral descriptors for deformable shapes. 2011;.

59. Litany O, Remez T, Rodolà E, Bronstein AM, Bronstein MM. Deep Functional Maps: Structured Prediction for Dense Shape Correspondence. 2017;

60. Donati N, Sharma A, Ovsjanikov M. Deep Geometric Functional Maps: Robust Feature Learning for Shape Correspondence. arXiv. 2020;.

61. Poulenard A, Ovsjanikov M. Multi-directional geodesic neural networks via equivariant convolution. ACM Transactions on Graphics (TOG). 2019;37(6):236. doi:10.1145/3272127.3275102.

62. Kipf TN, Welling M. Semi-Supervised Classification with Graph Convolutional Networks. 2016;.

63. Lei H, Akhtar N, Mian A. Spherical Kernel for Efficient Graph Convolution on 3D Point Clouds. IEEE Transactions on Pattern Analysis and Machine Intelligence. 2020;PP(99):1-1. doi:10.1109/tpami.2020.2983410.

64. Thomas H, Qi CR, Deschaud JE, Marcotegui B, Goulette F, Guibas LJ. KPConv: Flexible and Deformable Convolution for Point Clouds. arXiv. 2019;. 
65. Rosenberg S. The Laplacian on a Riemannian Manifold. In: The Laplacian on a Riemannian Manifold: An Introduction to Analysis on Manifolds. London Mathematical Society Student Texts. Cambridge University Press; 1997. p. 1-51.

66. Fey M, Lenssen JE. Fast Graph Representation Learning with PyTorch Geometric. In: ICLR Workshop on Representation Learning on Graphs and Manifolds; 2019.

67. Kingma DP, Ba J. Adam: A method for stochastic optimization. arXiv preprint arXiv:14126980. 2014;. 
(A)
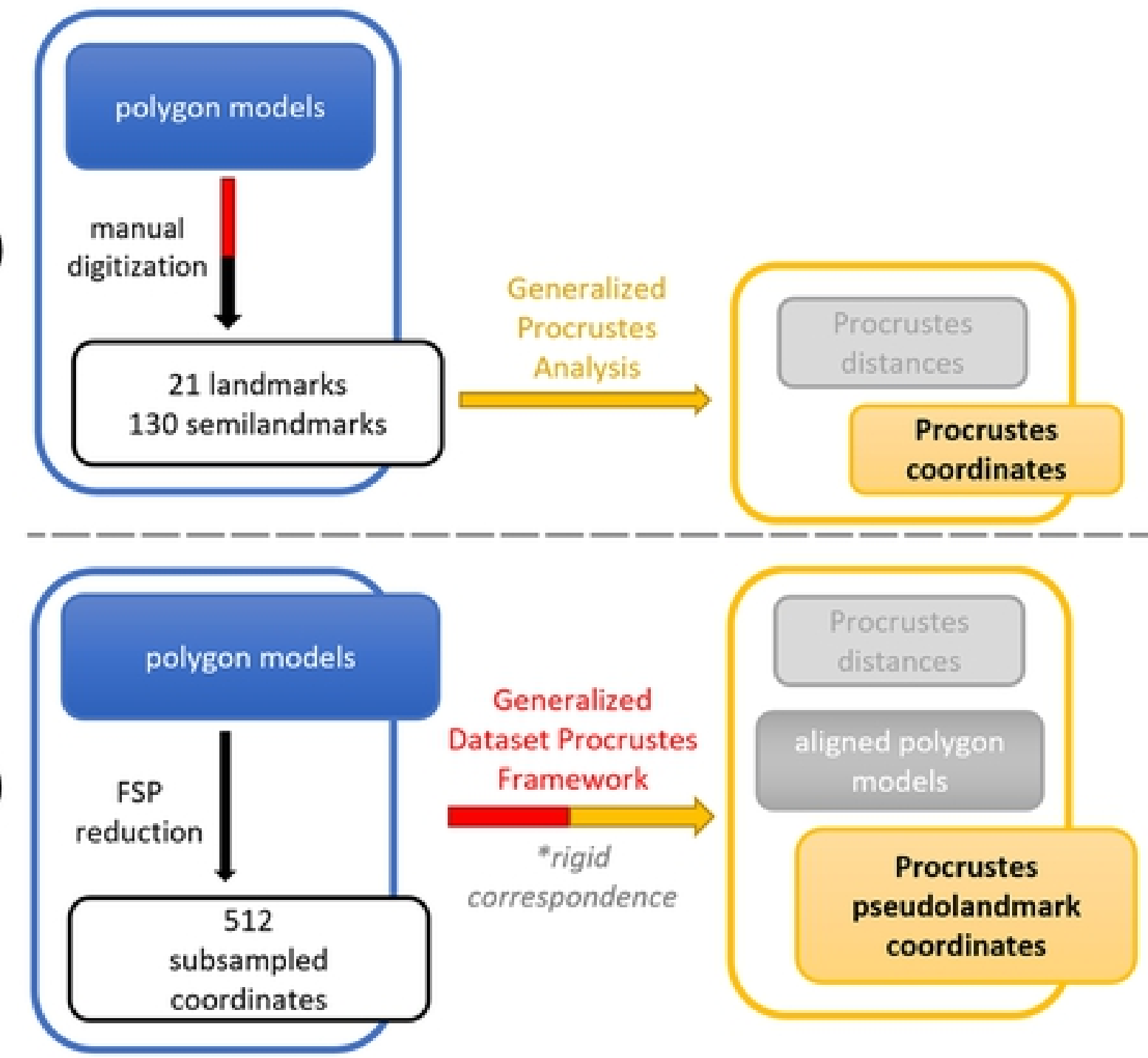

\section{Generalized}

(B)

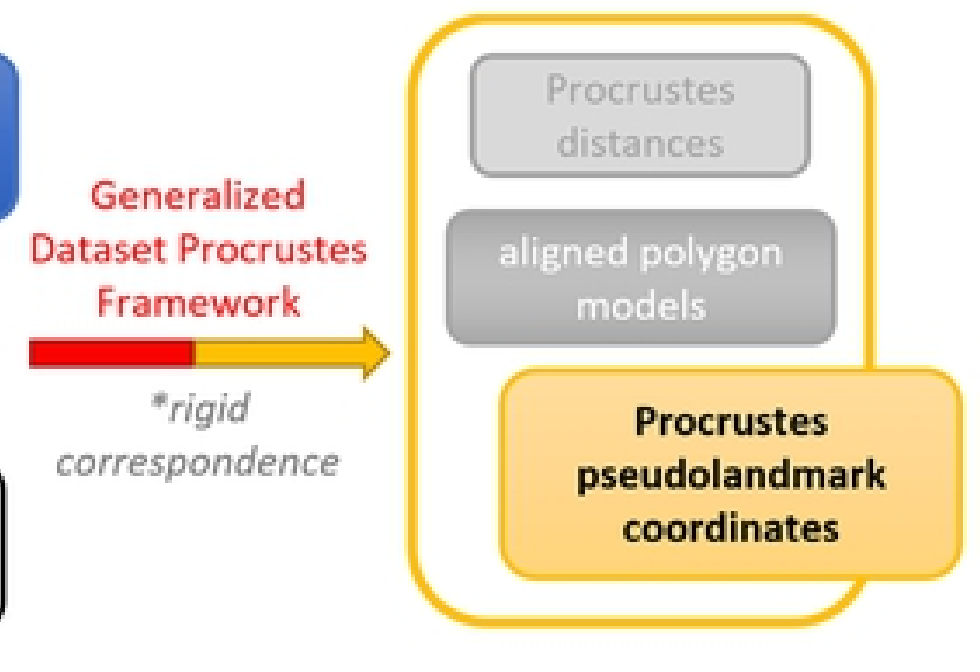

\section{Auto3DGM}

(C)
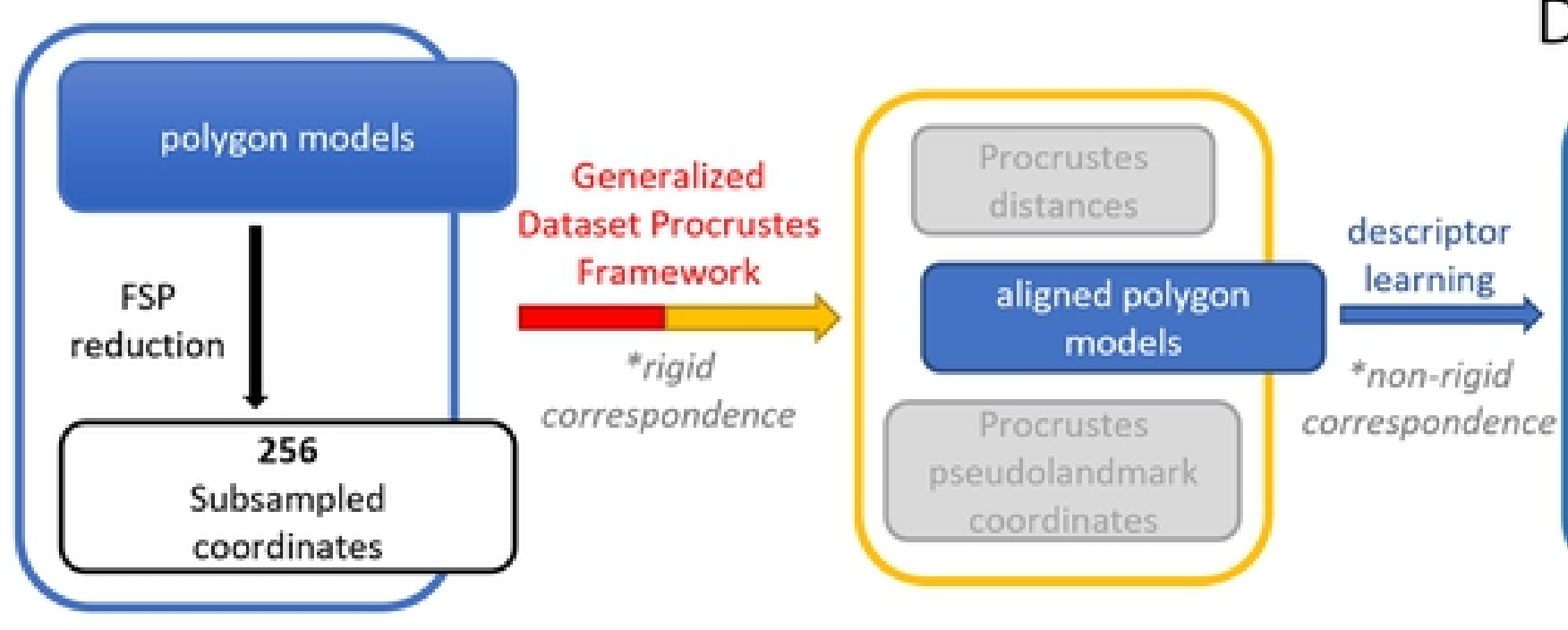

Descriptor Learning/FM Framework

Figure 1 


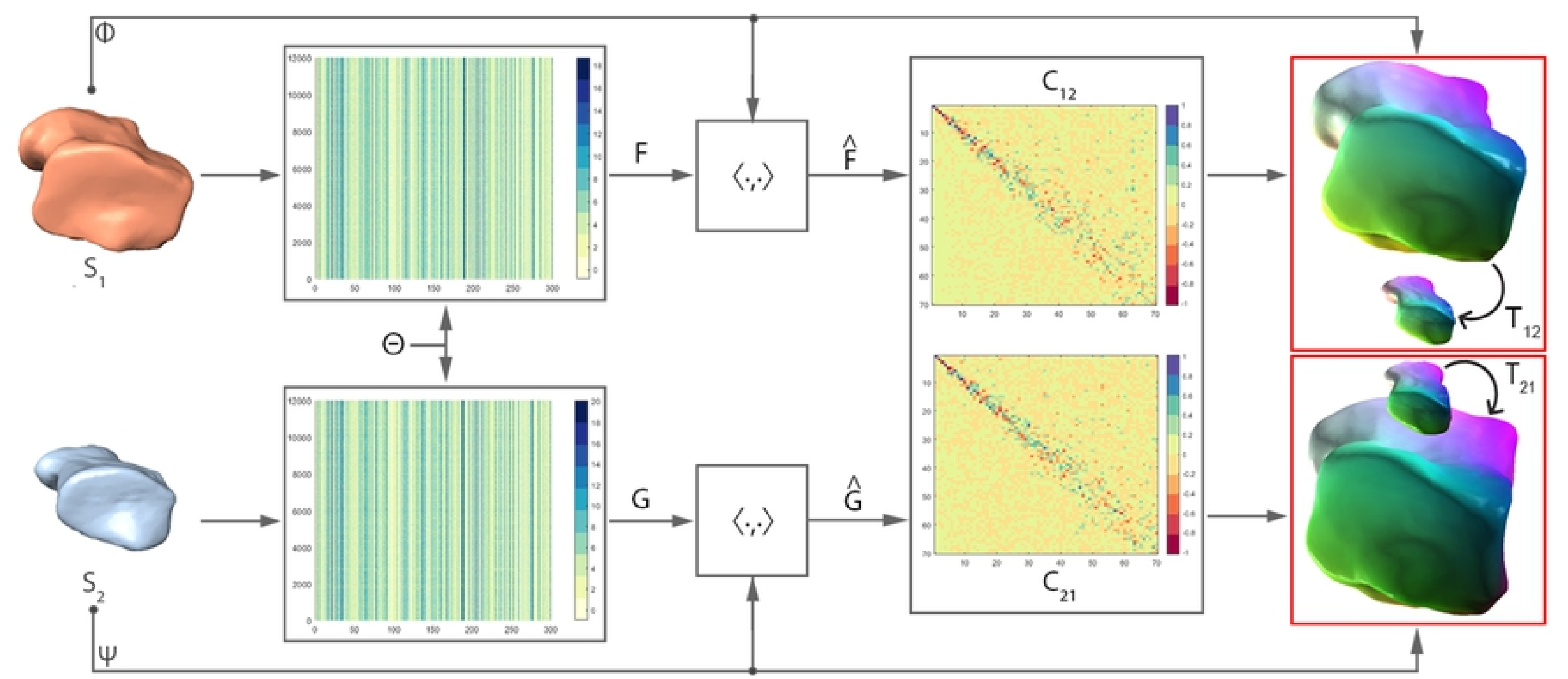

Figure 2 


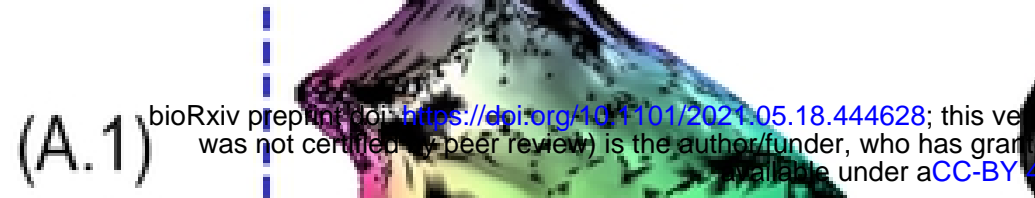

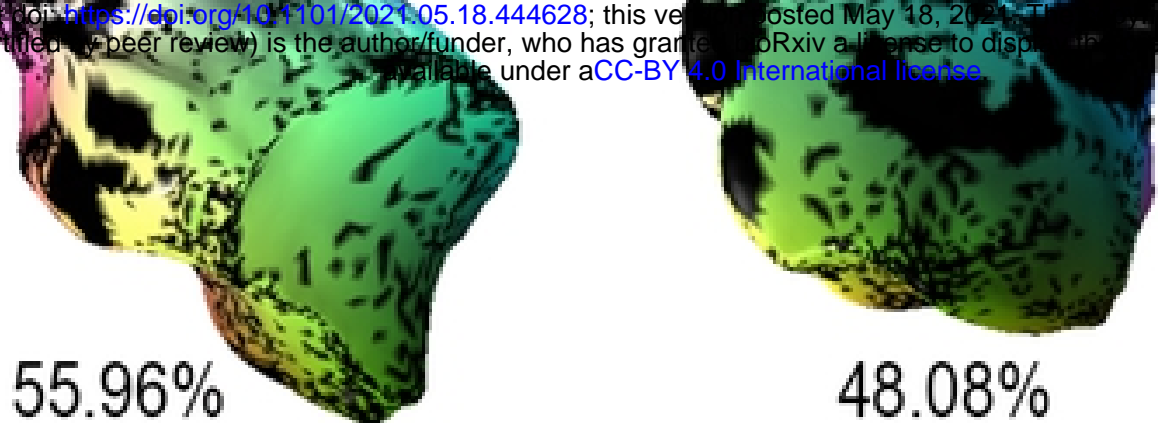

(A.2)

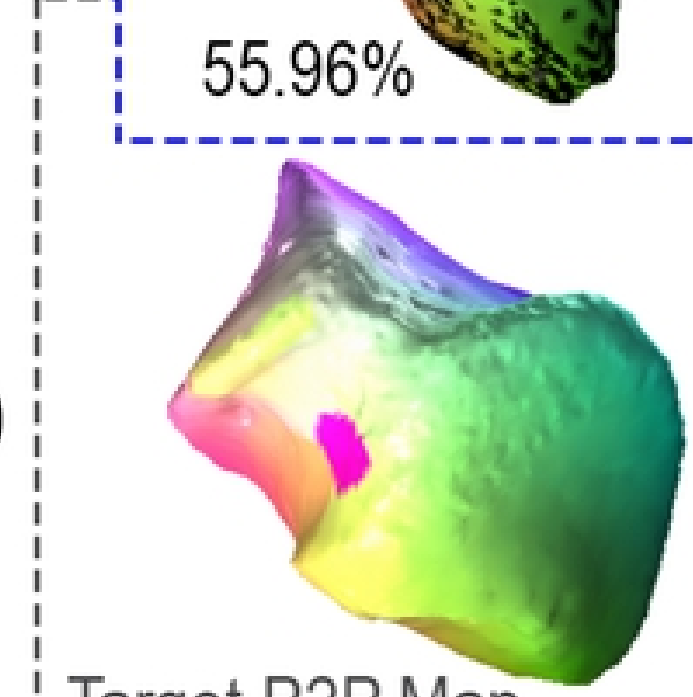

Target P2P Map

(B.1)

(B.2)
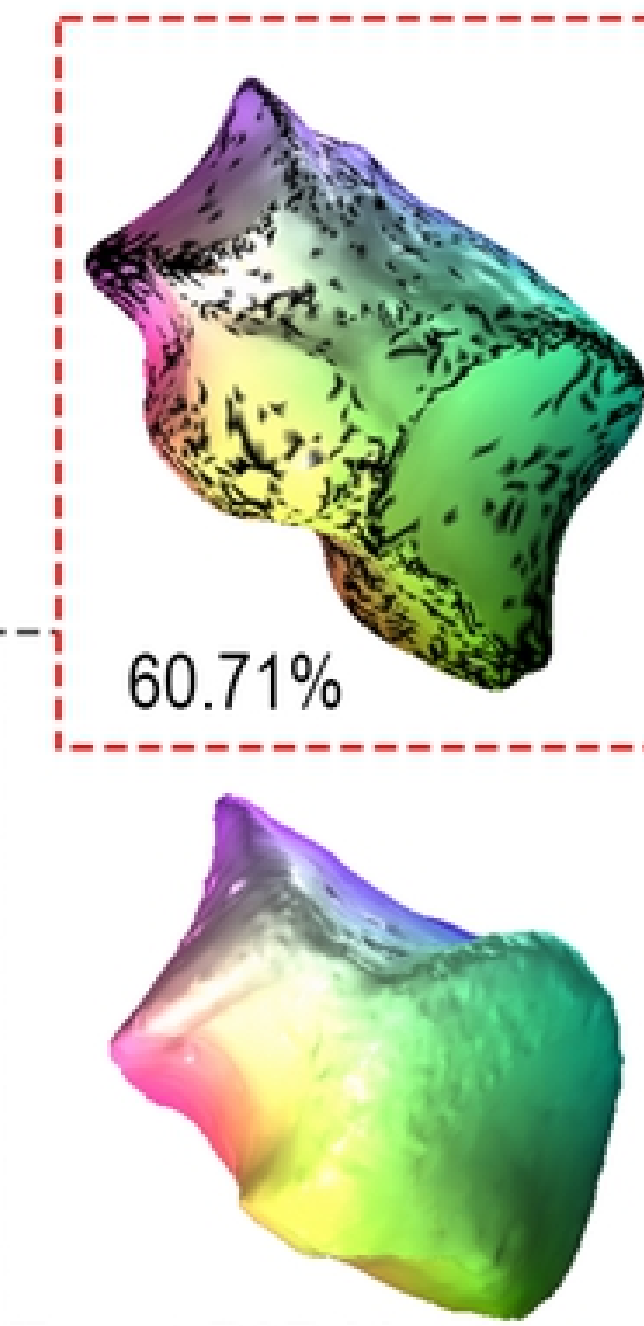

Target P2P Map
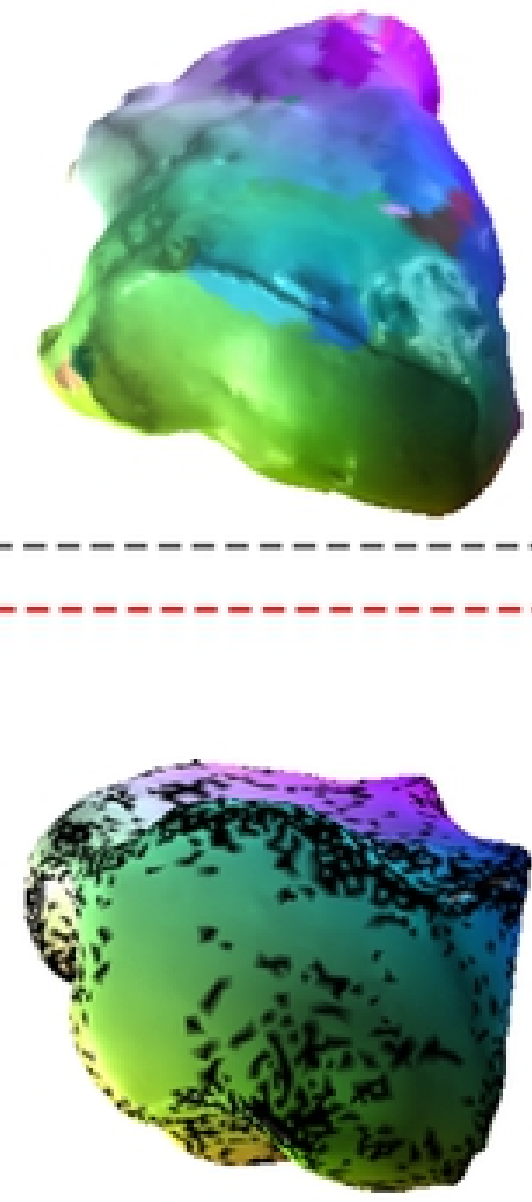

$60.60 \%$

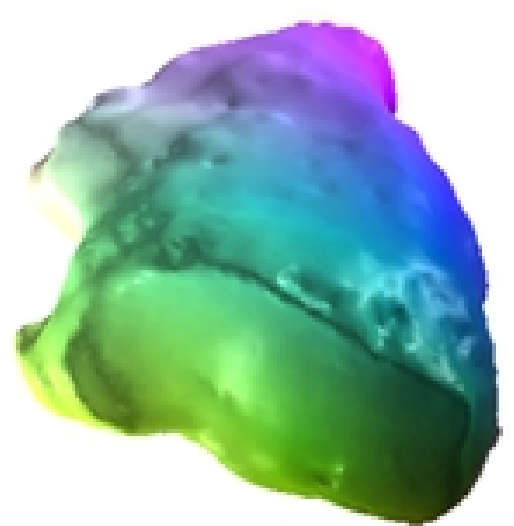

Initial Source P2P Coverage

ht holder for this prepr ${ }^{2}$ intrich
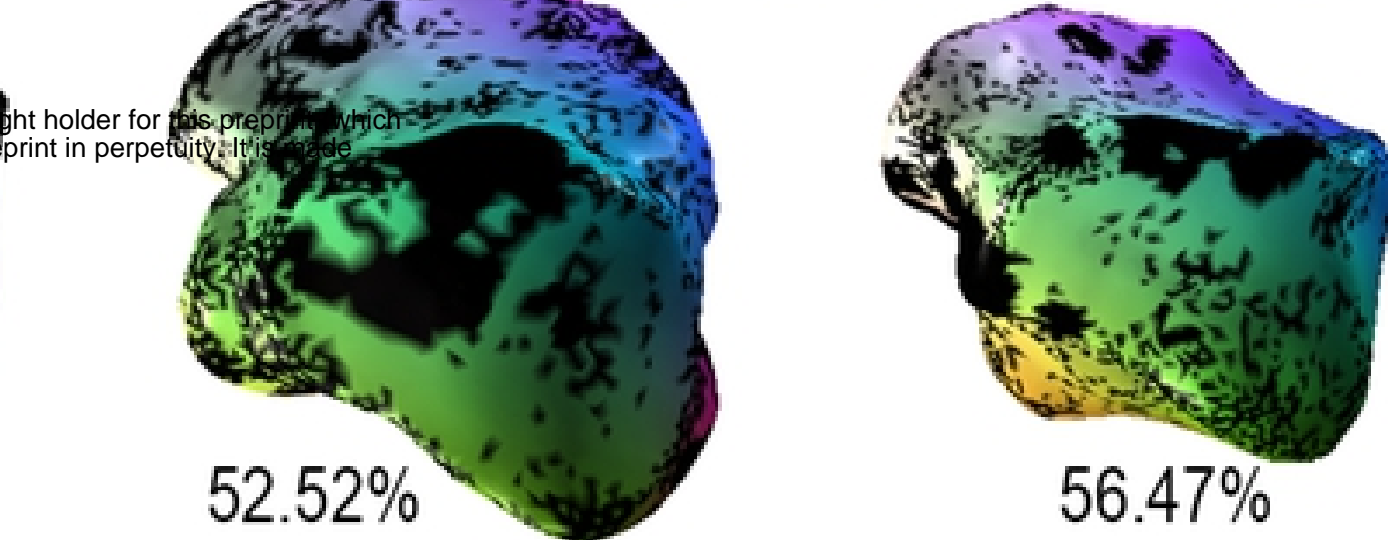

Refined Source $\mathrm{P} 2 \mathrm{P}$ Coverage
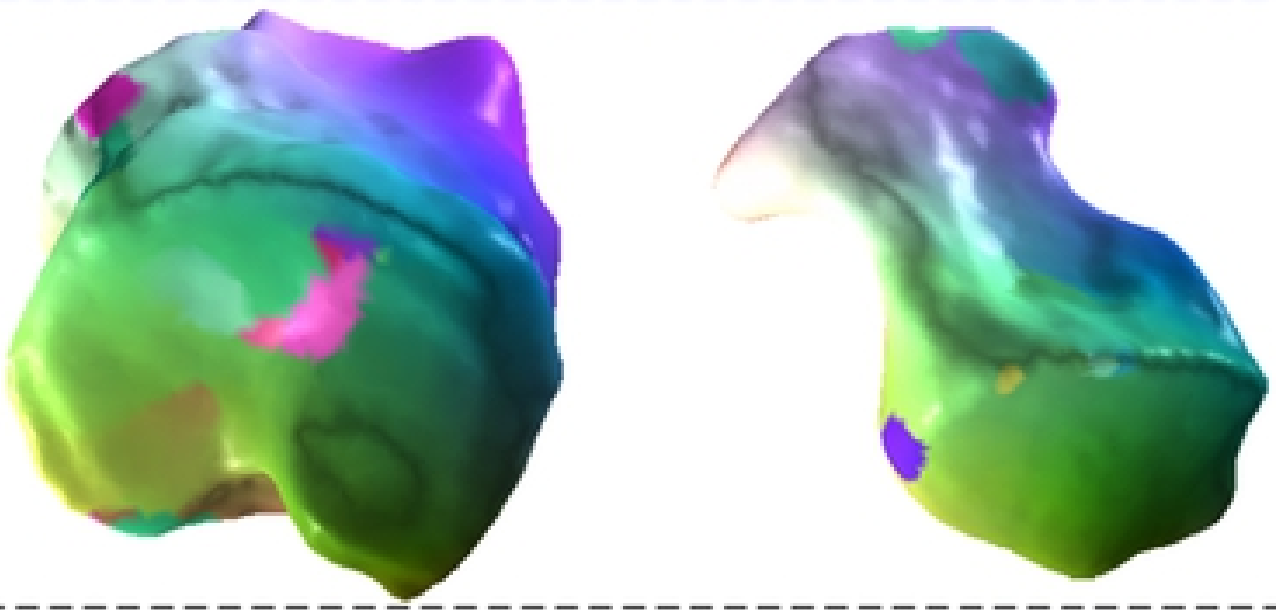

\section{Sag.}
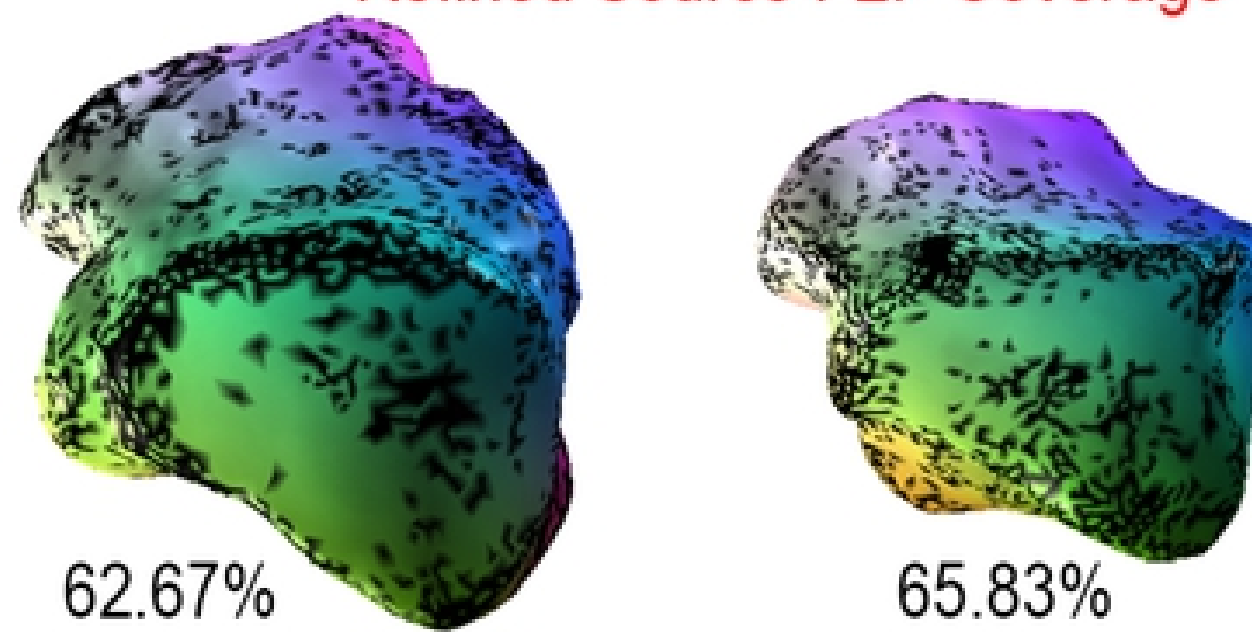

$65.83 \%$
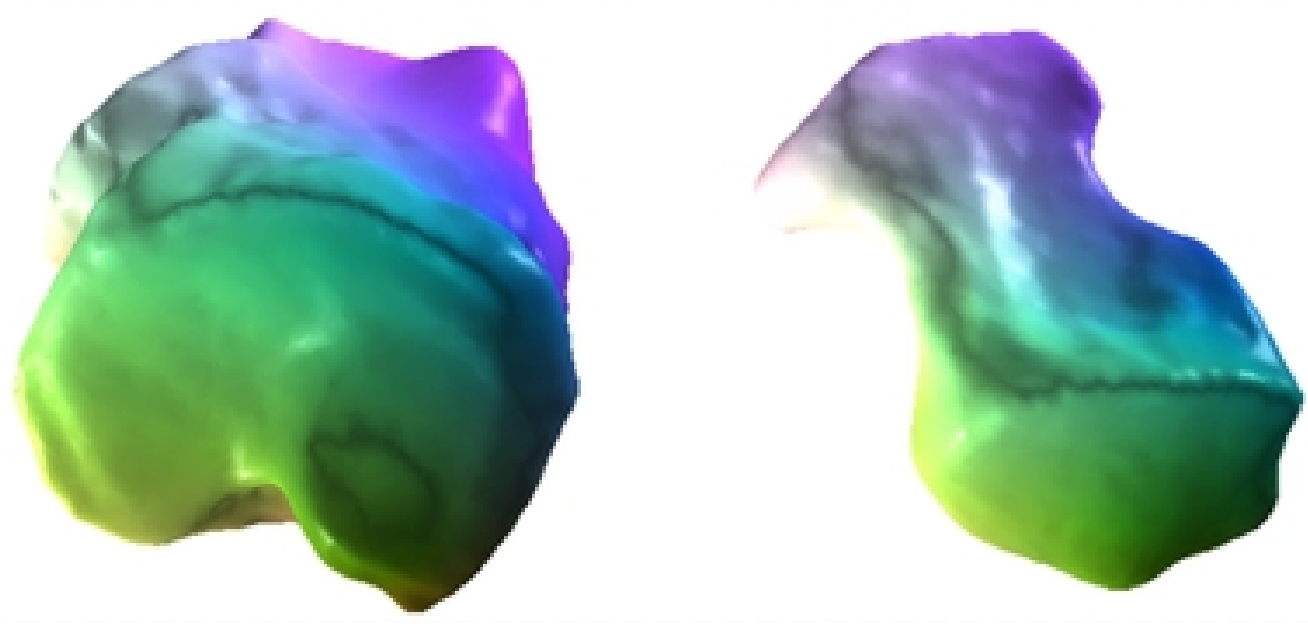

Figure 3 
(A)

21 Type I-III Homologous Landmarks
(B)

Proximal and Distal

Semilandmark

Patches

(130 landmarks)
(C)

Triangulated

Pseudolandmarks

(512 points)
(D)

Polygon Models

(12k+ vertices)
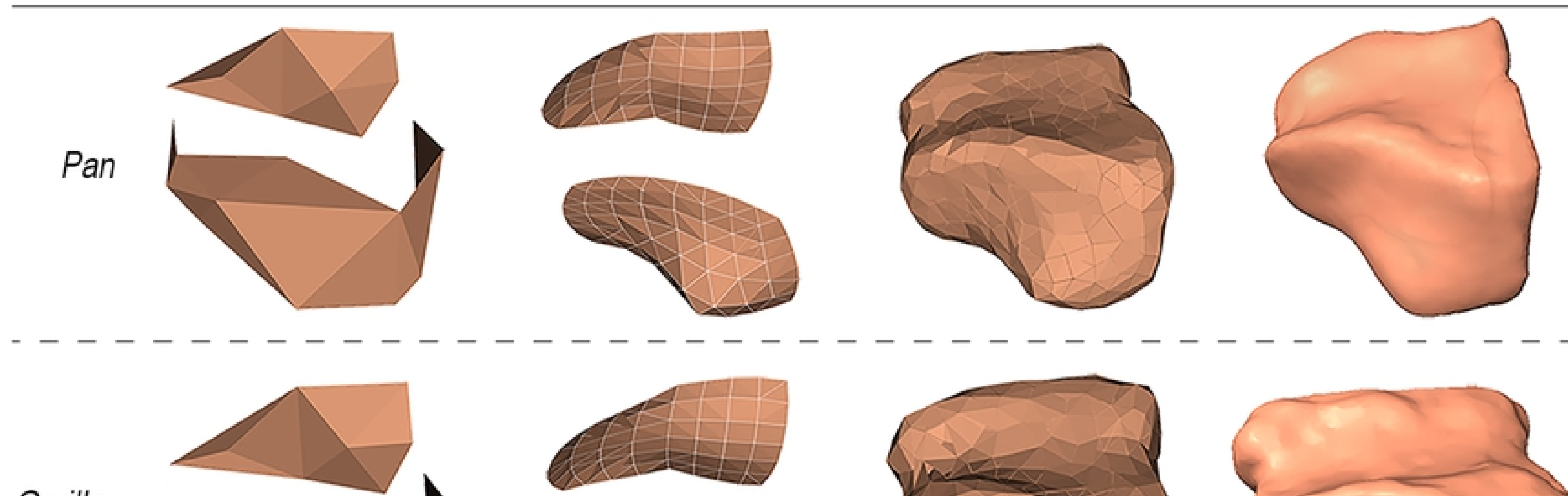

\section{Gorilla}

bioRxiv preprint doi: https://doi.org/10.1101/2021.05.18 44628; this version posted May 18, 2021. The copyright holder for this preprint (which was not cerl(fied by peer review) is the author/fund , who has granted bioRxiv a license to display the preprint in perpetuity.
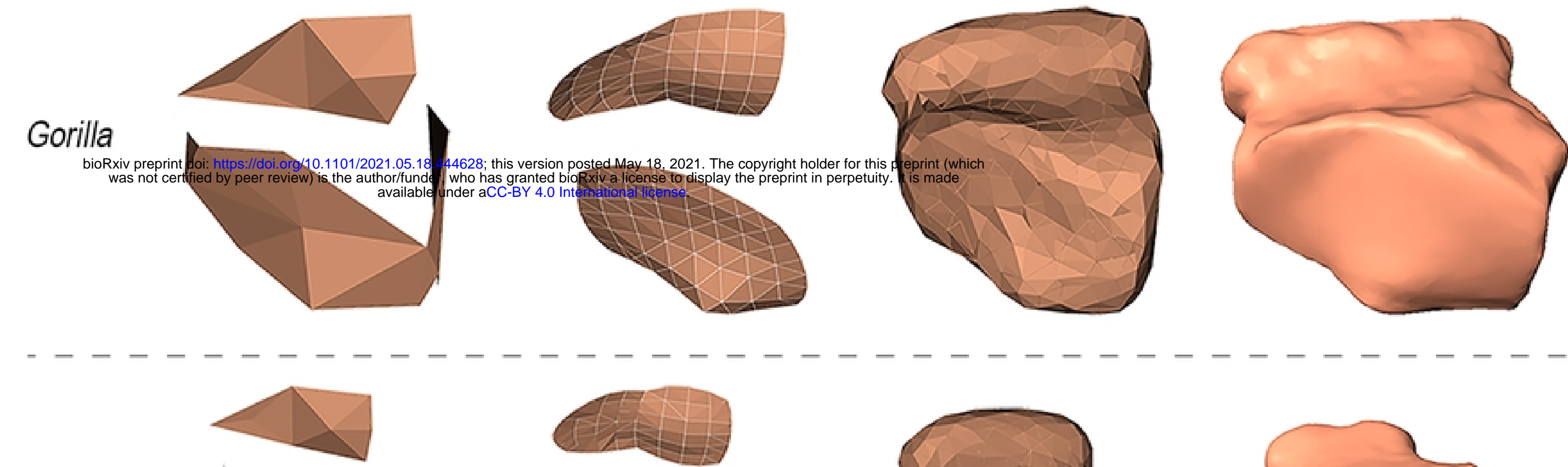

Hylobates
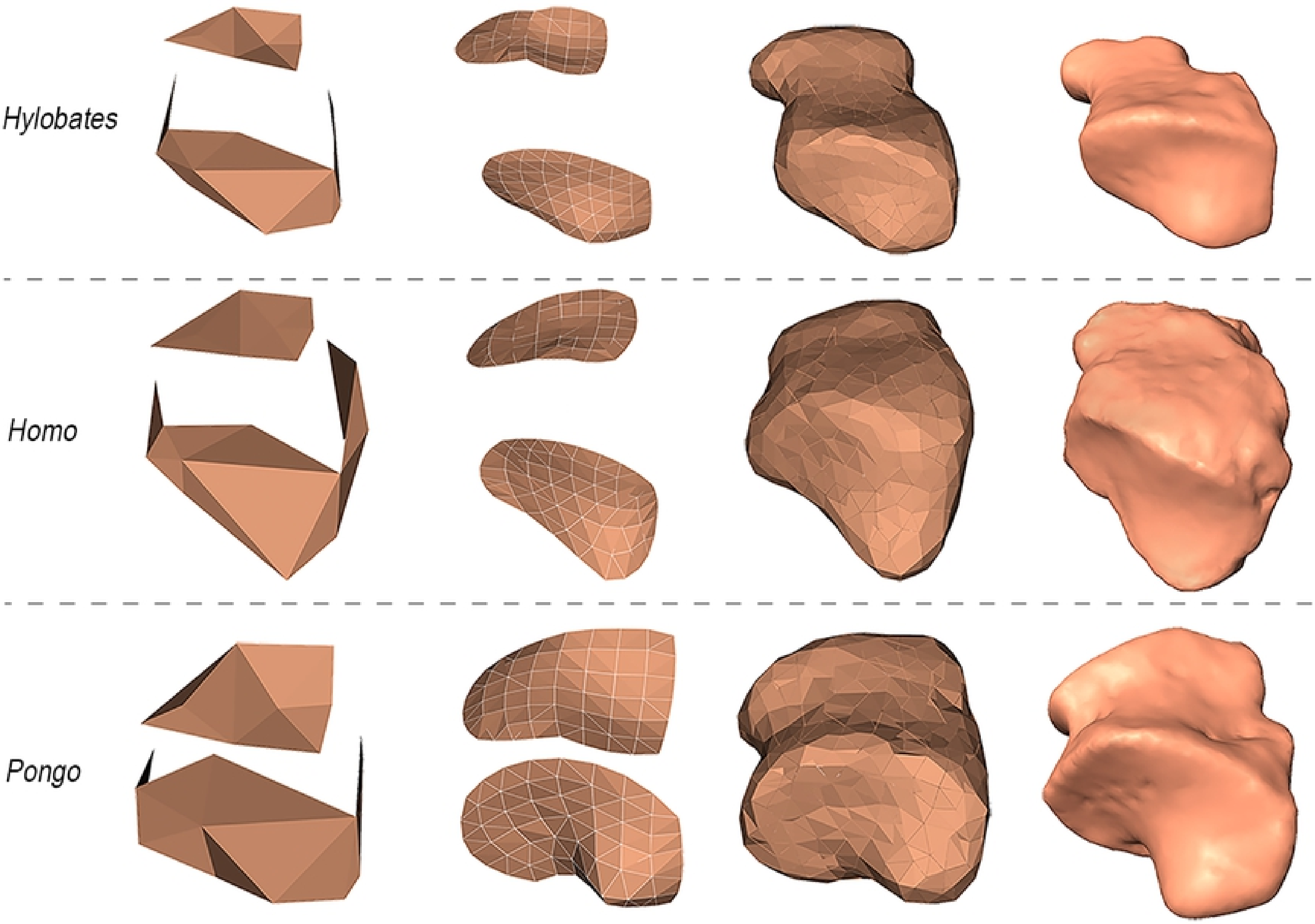

Figure 5 
Area-Based Weighted Distinctive Functions

(I)

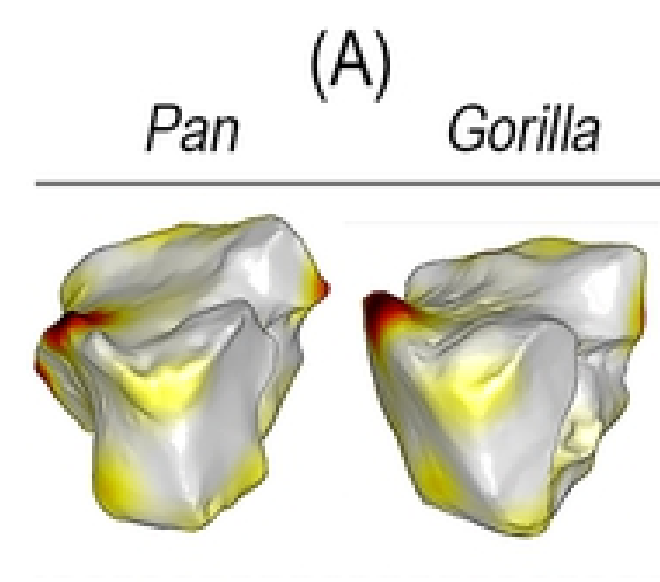

(II)

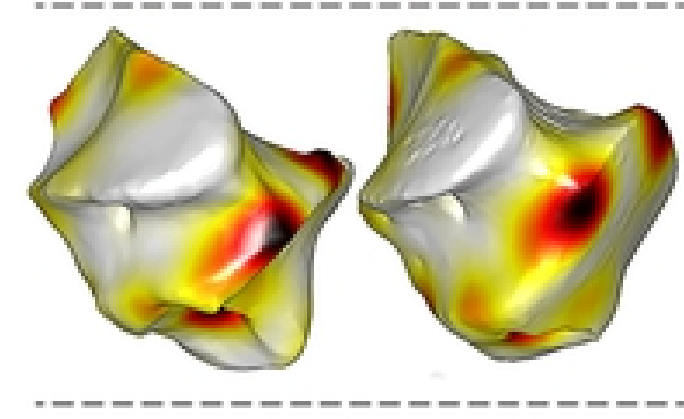

(III)

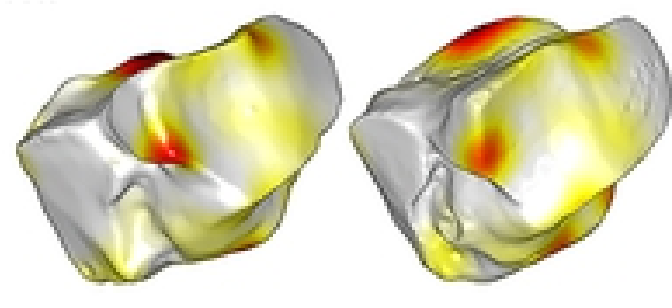

(IV)
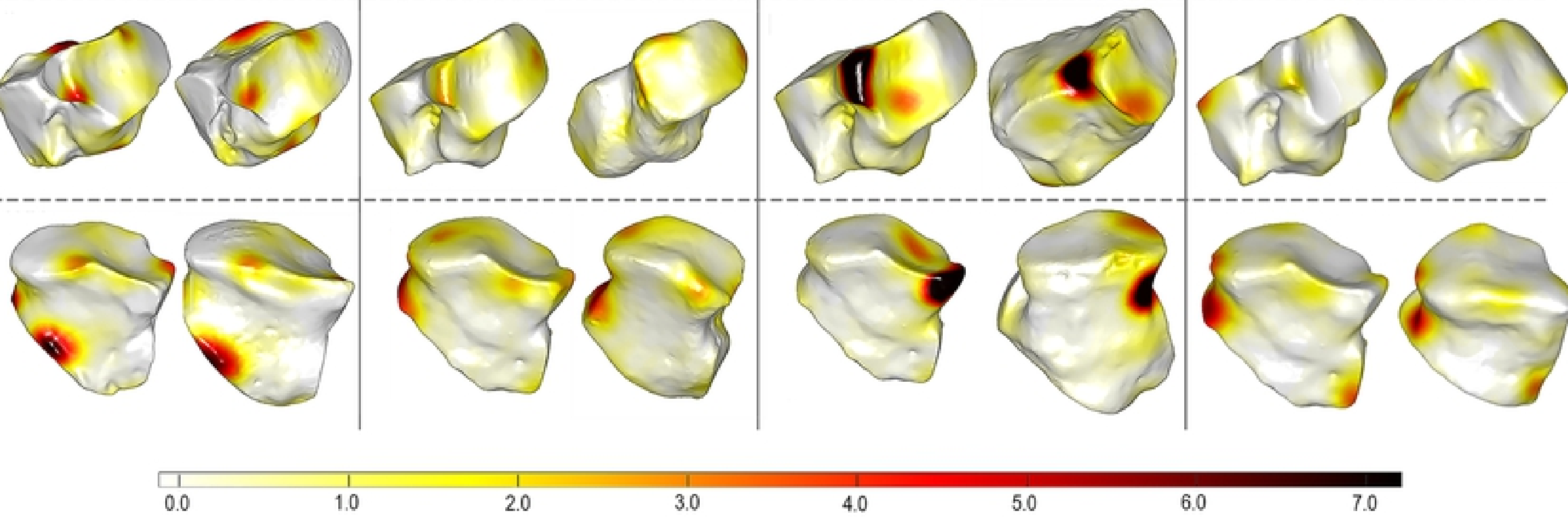

3.0

Figure 6 
Conformal Weighted Distinctive Functions

(I)

Pan ${ }^{(\mathrm{A})}$ Gorilla

(B)
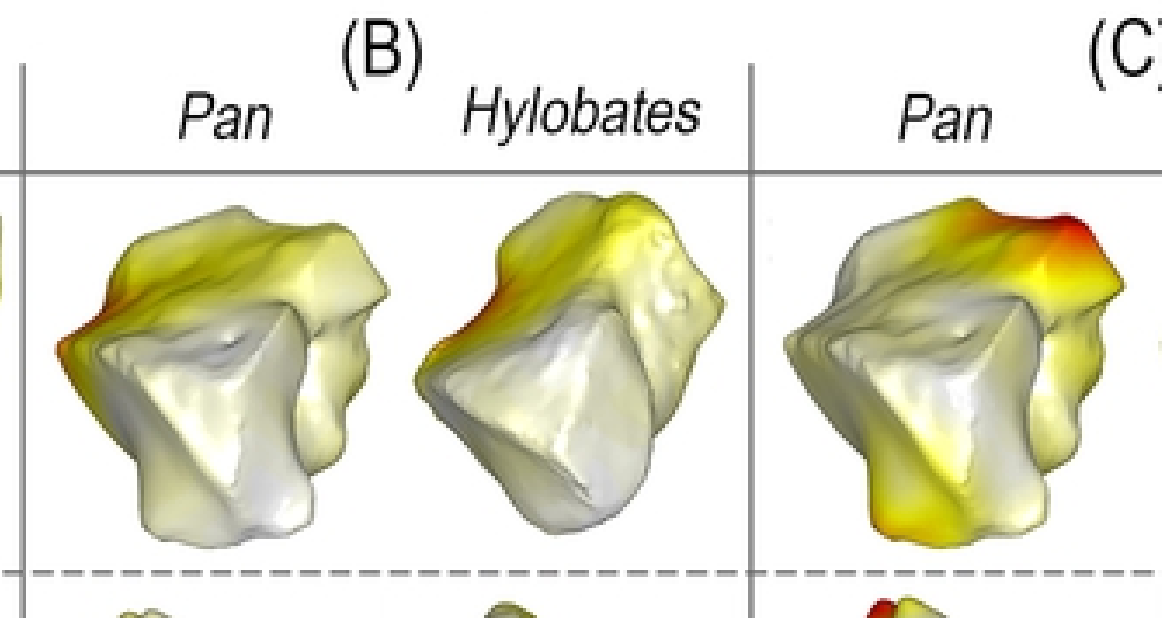

(C)

(D)

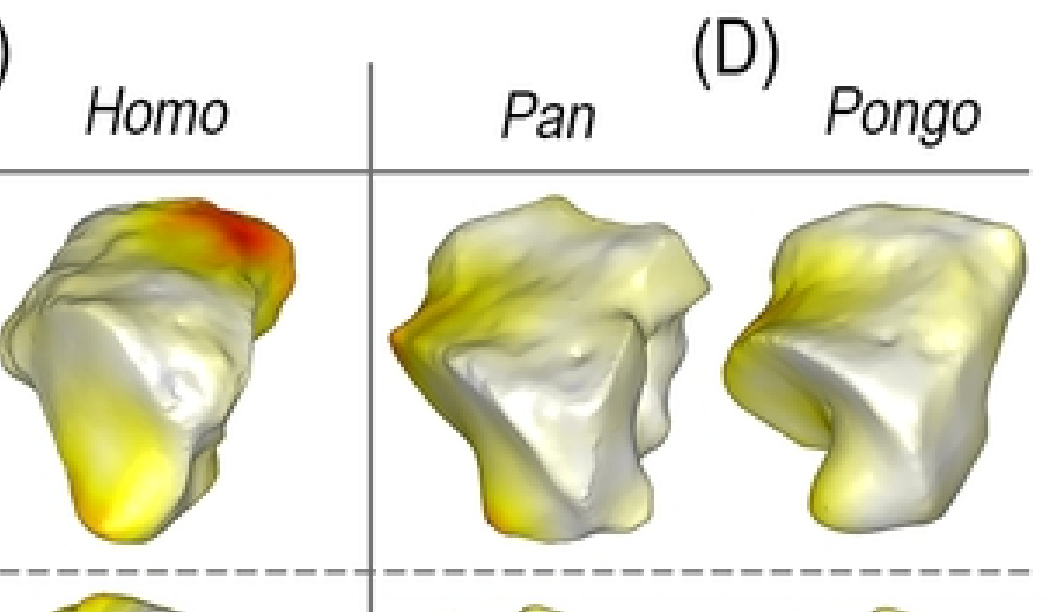

(II)
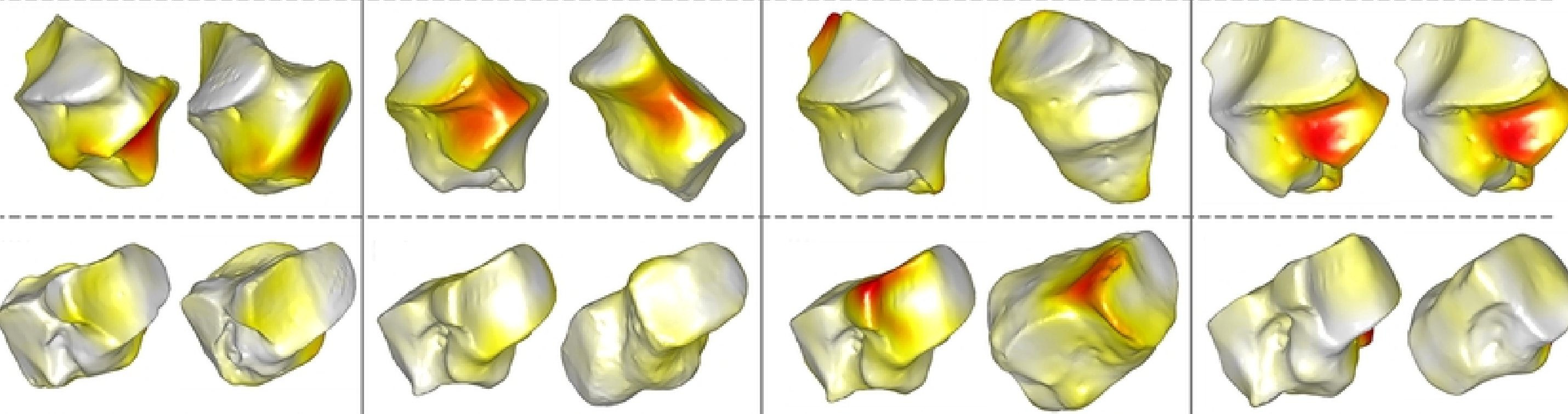

(III)
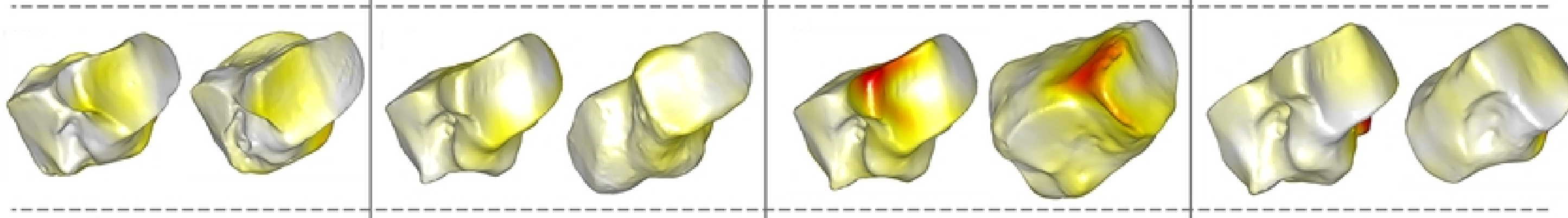

(IV)
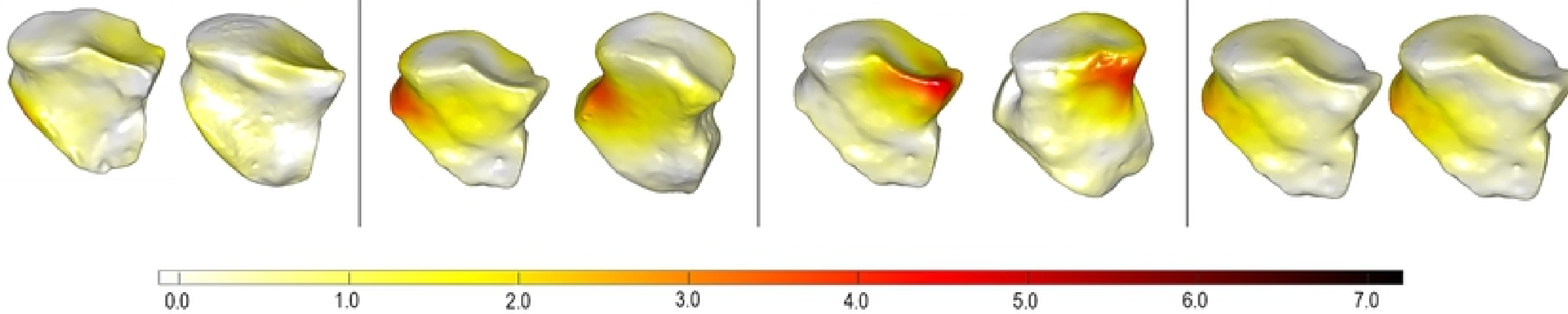

3.0

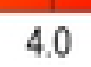

5.0

Figure 7 


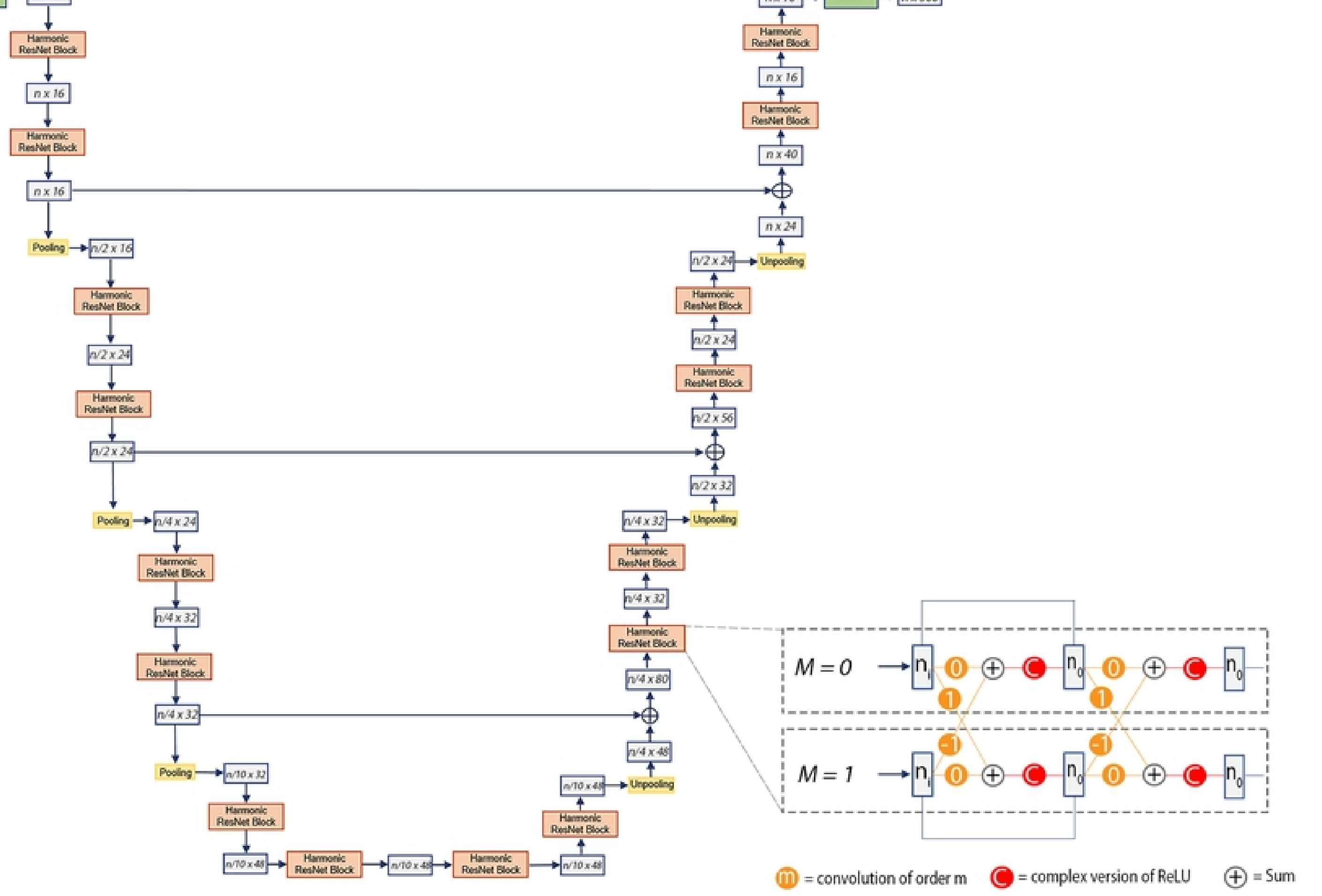




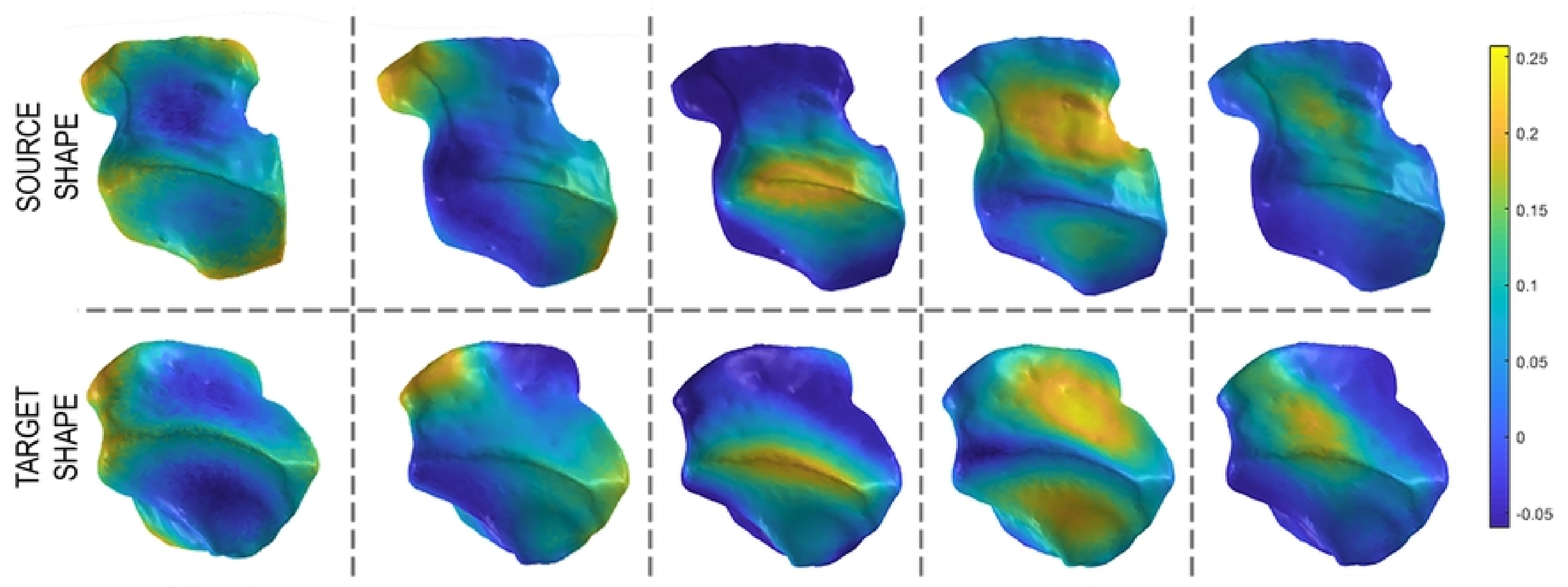

$\mathrm{S} 2 \mathrm{Fig}$ 


\section{(A) Pan source Hylobates target}

(B) Pan source Homo target
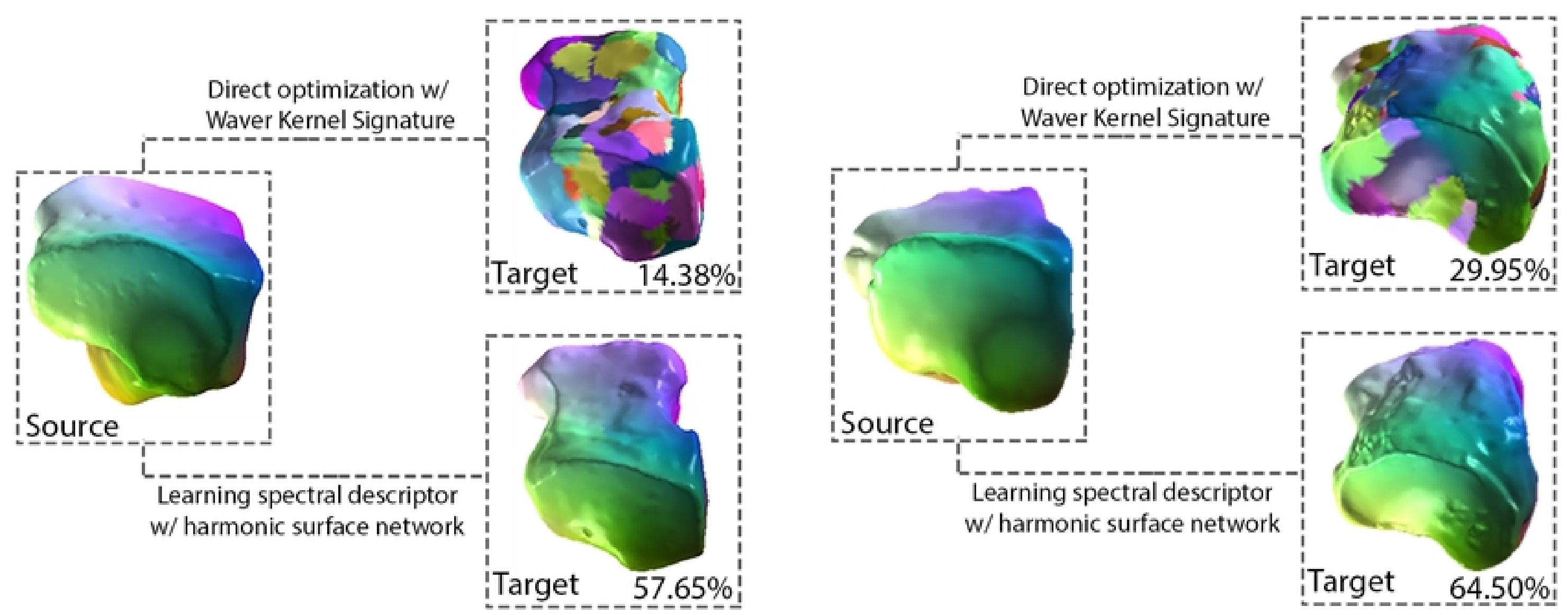


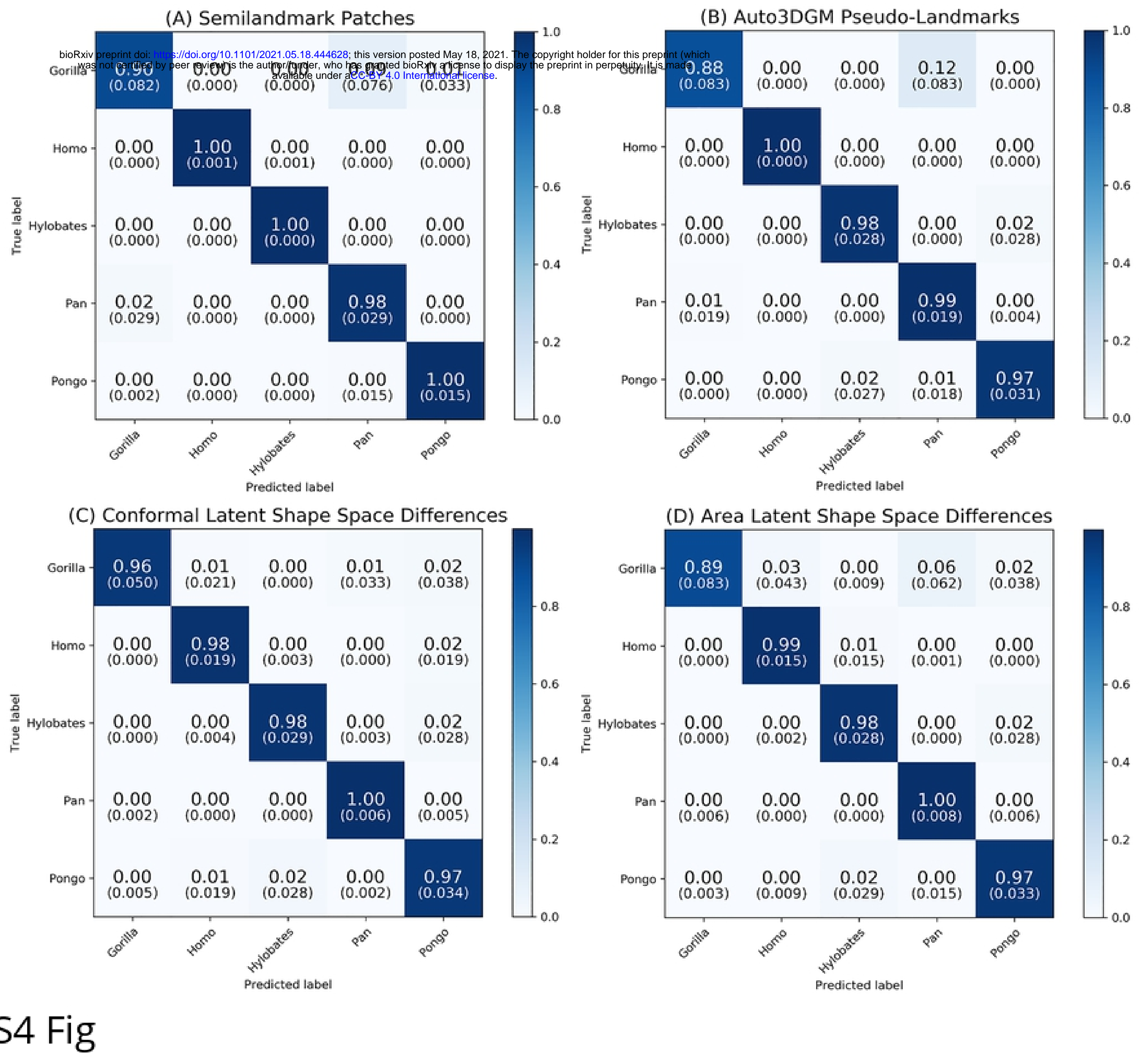

